\title{
A critical review of the mechanical properties of CoCrNi-based medium-entropy alloys
}

\author{
Dingfeng $\mathrm{Xu}$, Mingliang Wang, Tianxin Li, Xiangsai Wei, Yiping Lu
}

Key Laboratory of Solidification Control and Digital Preparation Technology (Liaoning Province), School of Materials Science and Engineering, Dalian University of Technology, Dalian 116024, Liaoning, China.

Correspondence to: Prof. Yiping Lu, Key Laboratory of Solidification Control and Digital Preparation Technology (Liaoning Province), School of Materials Science and Engineering, Dalian University of Technology, Dalian 116024, Liaoning, China. E-mail: luyiping@dlut.edu.cn; Dr. Mingliang Wang, Key Laboratory of Solidification Control and Digital Preparation Technology (Liaoning Province), School of Materials Science and Engineering, Dalian University of Technology, Dalian 116024, Liaoning, China. E-mail:wml_8778@163.com

How to cite this article: Xu D, Wang M, Li T, Wei X, Lu Y. A critical review of the mechanical properties of CoCrNi-based medium-entropy alloys. Microstructures 2022;2:2022001. https://dx.doi.org/10.20517/microstructures.2021.10

Received: 21 Nov 2021 First Decision: 13 Dec 2021 Revised: 20 Dec 2021 Accepted: 30 Dec 2021 Published: 13 Jan 2022

Academic Editor: Xiaozhou Liao Copy Editor: Xi-Jun Chen Production Editor: Xi-Jun Chen

\begin{abstract}
The CoCrFeMnNi alloy is one of the most notable first-generation high-entropy alloys and is also known as a Cantor alloy. This alloy was first proposed in 2004 and shows promising performance at cryogenic temperatures (CTs). Subsequent research has indicated that the equiatomic ternary CoCrNi medium-entropy alloy (MEA), as a subset of the Cantor alloy family, has better mechanical properties than the CoCrFeMnNi alloy. Interestingly, both the strength and ductility of the CoCrNi MEA are higher at CTs than at room temperature. CoCrNi-based alloys have attracted considerable attention in the metallic materials community and it is therefore important to generalize and summarize the latest progress in CoCrNi-based MEA research. The present review initially briefly introduces the discovery of the CoCrNi MEA. Subsequently, its tensile response and deformation mechanisms are summarized. In particular, the effects of parameters, such as critical resolved shear stress, stacking fault energy and short-range ordering, on the deformation behavior are discussed in detail. The methods for strengthening the CoCrNi MEA are then reviewed and divided into two categories, namely, modifying microstructures and adjusting chemical compositions. In addition, the mechanical performance of CoCrNi-based MEAs, including their dynamic shear properties, creep behavior and fracture toughness, is also deliberated. Finally, the development prospects of CoCrNi-based MEAs are proposed.
\end{abstract}


Keywords: Medium-entropy alloys, mechanical properties, deformation mechanism, strengthening mechanism

\section{INTRODUCTION}

The traditional alloy design strategy takes one element as the principal constituent and adds other minor elements to optimize the properties. However, the development of conventional alloys is approaching its limits. Since Cantor et al. ${ }^{[1]}$ and Yeh et al. ${ }^{[2]}$ proposed the concept of high-entropy alloys (HEAs) in 2004, this new alloy design strategy has attracted extensive attention. This strategy theoretically provides almost limitless possibilities for the design of the composition and optimization of alloy properties. HEAs are known to exhibit superior physical, chemical and mechanical properties compared to conventional alloys, including excellent thermal stability, radiation resistance, corrosion resistance and ultrahigh fracture toughness ${ }^{[3-8]}$.

\section{Discovery of CoCrNi alloy}

Over a development process of nearly 20 years, several typical HEA systems have been developed and studied in detail. One of the most classic HEAs is $\mathrm{CoCrFeMnNi}$ with a single-phase face-centered cubic (FCC) structure. This alloy displays an excellent fracture toughness in cryogenic temperature (CT) tensile tests, which exceeds $200 \mathrm{MPa} \cdot \mathrm{m}^{1 / 2}$ for crack initiation and rises to $>300 \mathrm{MPa} \cdot \mathrm{m}^{1 / 2}$ for stable crack growth. The toughness levels of the alloy are comparable to those of the best cryogenic steels ${ }^{[6]}$.

However, Otto et al. ${ }^{[9]}$ found that most of the HEAs discussed in the literature actually appear to be multiphase alloys ${ }^{[10-19]}$ and their configurational entropies should be low rather than high. Only a few multielement equiatomic alloys are truly single-phase solid solutions. Therefore, the number of alloy components is not the most important factor and the chemical nature of the components should also be studied critically.

Wu et al. ${ }^{[20]}$ investigated the lower-order systems of the CoCrFeMnNi alloy that formed solid solution alloys consisting of a single FCC phase. Using X-ray diffraction and scanning electron microscopy, it was found that three of the five possible quaternaries ( $\mathrm{CoCrFeNi}$, CoFeMnNi and $\mathrm{CoCrMnNi}$ ), five of the ten possible ternaries (CoFeNi, $\mathrm{CrFeNi}$, FeMnNi, $\mathrm{CoCrNi}$ and $\mathrm{CoMnNi}$ ) and two of the possible ten binaries (FeNi and $\mathrm{CoNi}$ ) were single FCC structures. An investigation of the microhardness evolution as a function of heat treatment temperature after cold rolling was also performed [Figure 1]. The ternary alloy CoCrNi was harder than the other alloys, suggesting that the number of elements cannot solely determine the solid solution hardening phenomenon in multi-component alloys.

In another work, Wu et al. ${ }^{[21]}$ investigated the effect of temperature on the tensile properties in the temperature range of 77-673 K [Figures 2 and 3], wherein all the alloys were subsets of the CoCrFeMnNi HEAs.

From Figures 2 and 3, it can be found that CoCrNi exhibits the highest YS and extent of work hardening at 77-673 K, indicating that the nature of the component elements must be considered when designing alloys.

The CoCrNi alloy is particularly noteworthy given its excellent performance and has been the subject of many studies. In the next section, we briefly explain the development of the CoCrNi MEA. 


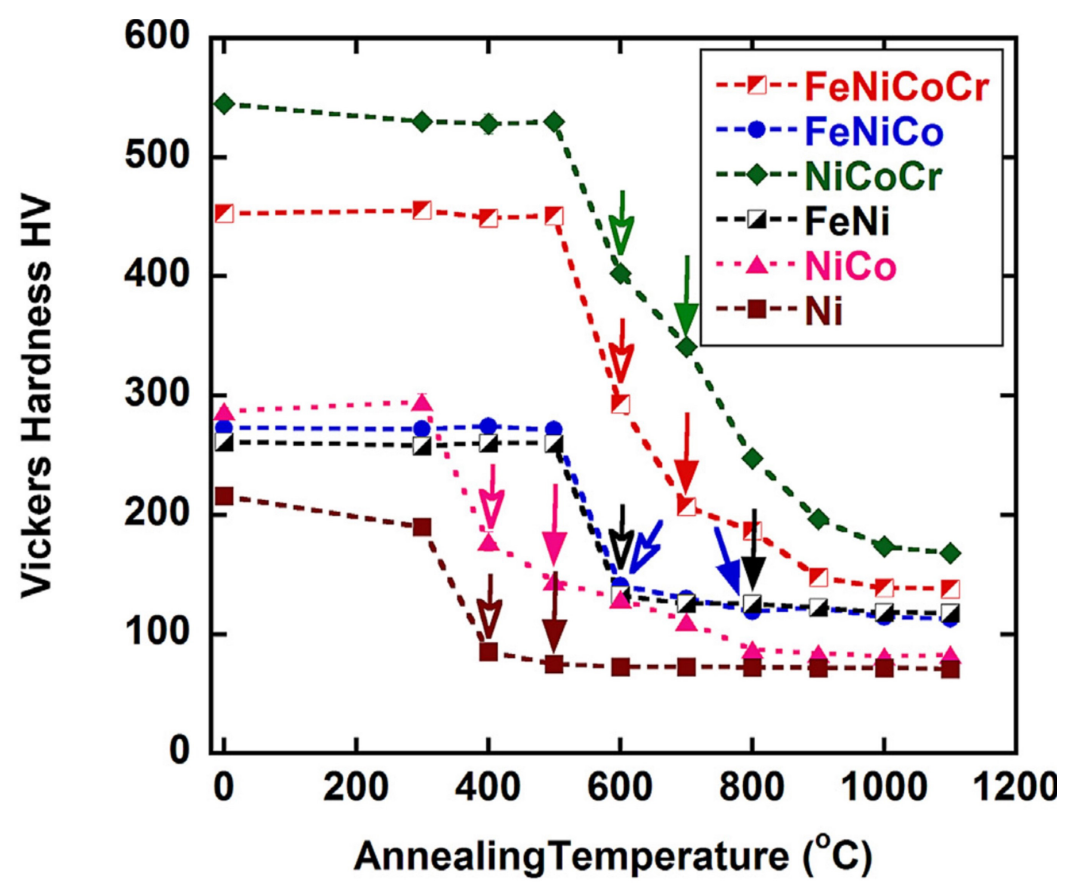

Figure 1. Microhardness of equiatomic alloys and $\mathrm{Ni}$ after rolling and subsequent annealing at various temperatures for $1 \mathrm{~h}$. Open and closed arrows represent the approximate start and finish of recrystallization, respectively ${ }^{[20]}$.

\section{Development of CoCrNi alloy}

CoCrNi has been extensively studied since 2014. From 2014 to 2017, the focus was mainly on the equiatomic ternary alloy. Initially, the ternary phase diagram, atomic diffusion behavior and quantum critical behavior of the $\mathrm{CoCrNi}$ alloy were studied ${ }^{[22-26]}$. The deformation mechanisms at room temperature (RT) and CTs were revealed ${ }^{[27-30]}$ and the mechanism of radiation damage reduction was proposed ${ }^{[31]}$. Laplanche et al. ${ }^{[32]}$ explored the reasons for the superior properties of the CoCrNi alloy compared to the CoCrFeMnNi alloy. Research into the short-range order (SRO) of CoCrNi was reported as early as $2017^{[33]}$ and it showed that it can play a vital role in lowering the electrical and thermal conductivities in the alloy.

Since 2018, the number of reports on the CoCrNi alloy has increased dramatically. The understanding of various aspects of the alloy has been gradually deepened, including lattice distortion (LD) ${ }^{[34-36]}$, stacking fault energy (SFE) ${ }^{[37-40]}$ and the critical shear stress of dislocations and twins ${ }^{[41]}$. The dislocation, twinning, phase transformation and even texture during deformation have been systematically studied ${ }^{[42-47]}$. In overcoming the trade-off between strength and ductility, various factors, including grain refinement ${ }^{[48,49]}$, heterogeneous structure $^{[50,51]}$ and alloying ${ }^{[52-61]}$, have been studied. In addition to their RT and CT properties, their high temperature creep ${ }^{[62]}$ and oxidation resistance ${ }^{[63]}$ has also been explored. In addition to quasi-static tensile tests, dynamic shear ${ }^{[6]}$ and impact ${ }^{[65]}$ experiments have also been performed.

\section{TENSILE RESPONSE AND TEXTURAL EVOLUTION}

\section{Tensile response}

Figure 4 shows the tensile response of the CoCrNi alloy under tensile testing at RT and CTs. The alloy showed a superior strength and ductility at CTs than at RT. At all strain levels, the strain hardening rate in cryogenic conditions was higher than at $\mathrm{RT}^{[28]}$. 

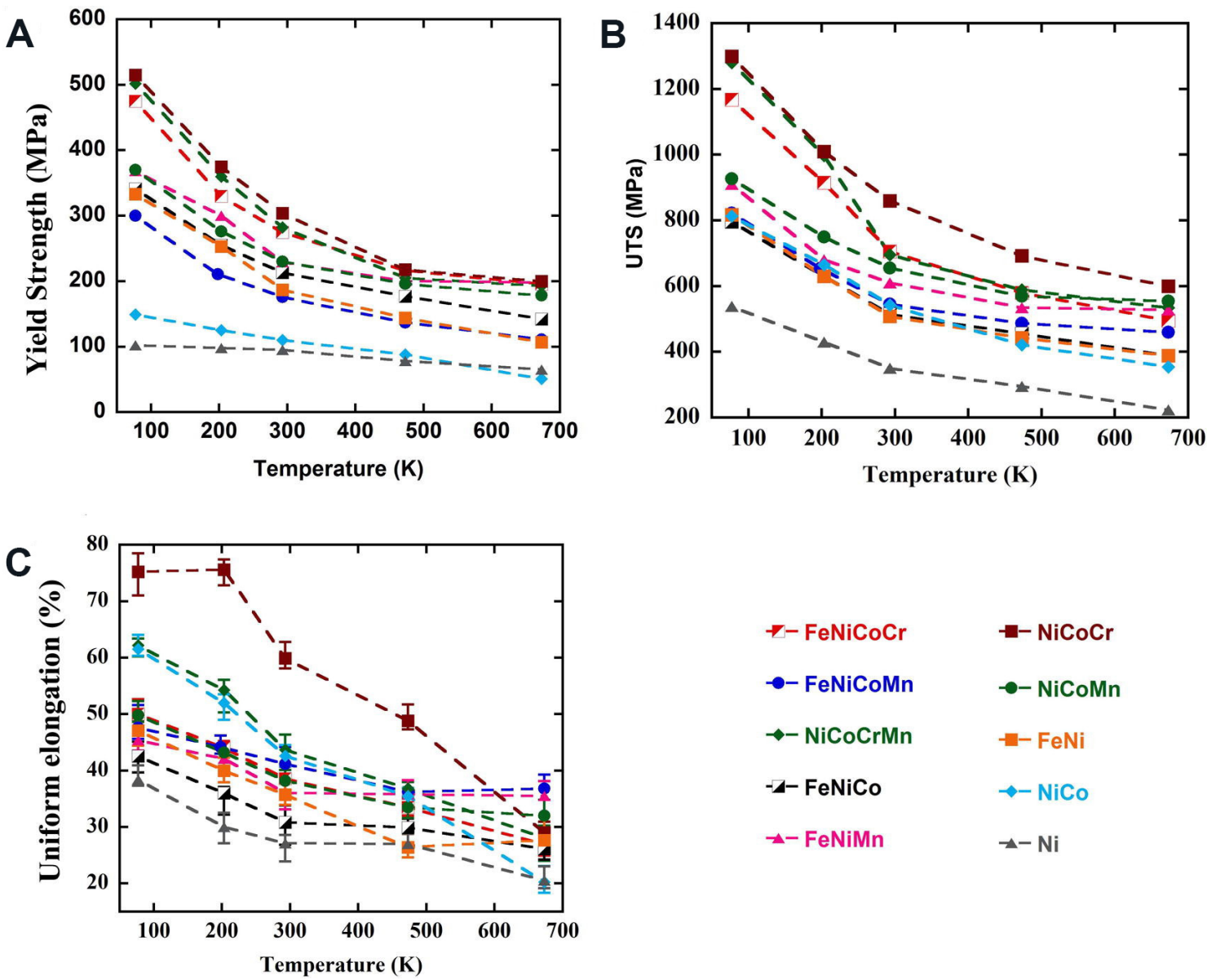

Figure 2. Temperature dependence of (A) yield stress (YS), (B) ultimate tensile stress (UTS) and (C) uniform elongation to fracture for equiatomic alloys and $\mathrm{Ni}^{[21]}$.

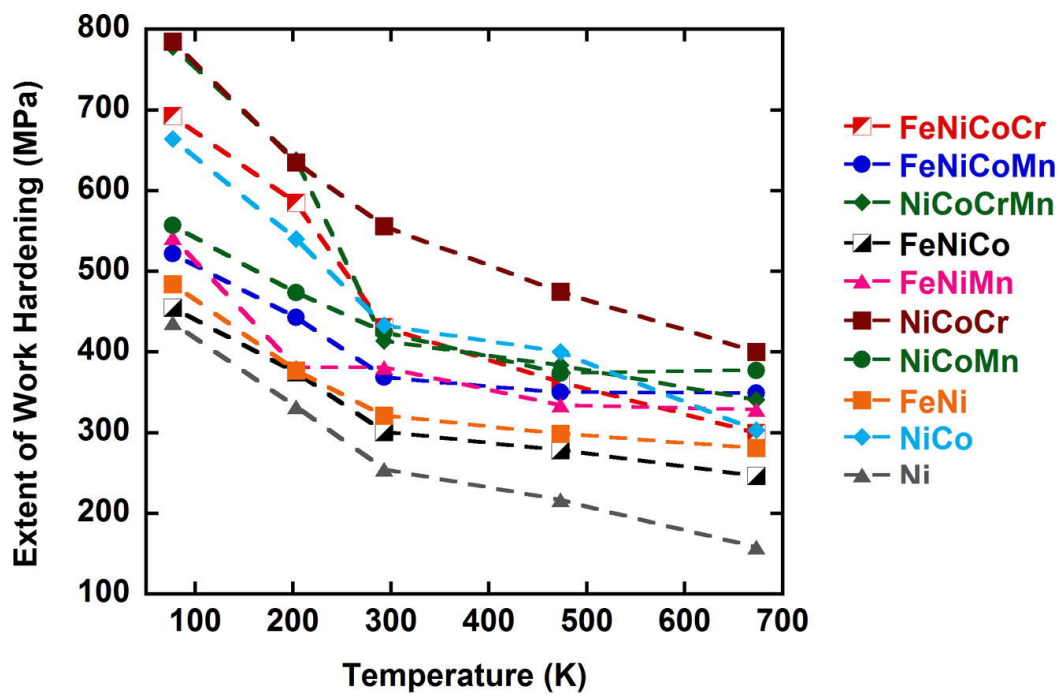

Figure 3. Effect of temperature on extent of work hardening (UTS-YS) for equiatomic alloys and $\mathrm{Ni}^{[21]}$. 


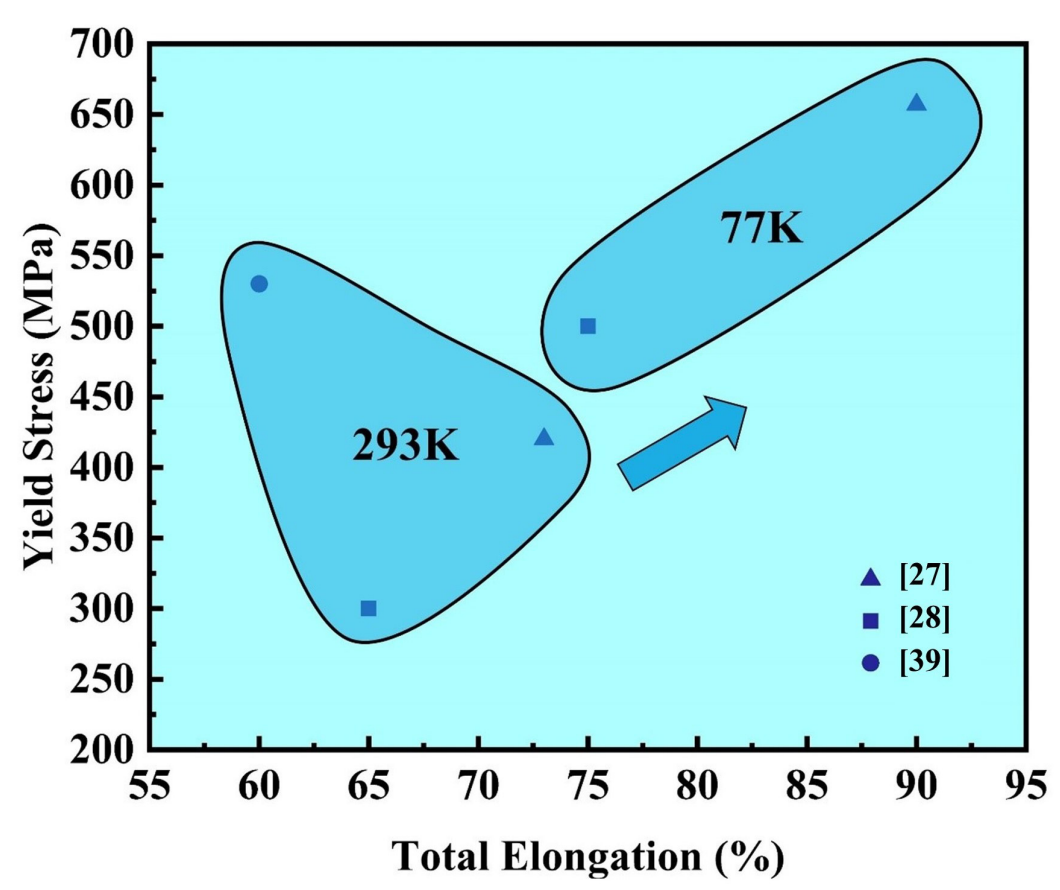

Figure 4. Tensile response of equiatomic ternary CoCrNi alloy at different temperatures ${ }^{[27,28,39]}$.

The excellent mechanical properties of the CoCrNi alloy are strongly dependent on the orientation. The uniaxial tensile responses of three orientations ([111], [110] and [123]) were investigated [Figure 5] $]^{[66]}$. At the early stage of deformation, the single slip dominated in the [123] orientation. Subsequently, multiple slip and nano-twinning were prominent in the [111] orientation. Nano-twinning prevailed as early as $4 \%$ strain along the $[110]$ orientation.

\section{Factors affecting tensile response}

\section{Critical resolved shear stress}

Abuzaid et al. ${ }^{[6]}$ quantitatively measured the critical resolved shear stress (CRSS) for the slip of the equiatomic CoCrNi alloy at RT and CTs. The slip CRSS is temperature dependent. As the temperature decreases, the slip CRSS increases. It was found to be $78 \mathrm{MPa}$ at $298 \mathrm{~K}$ and between 140 and $160 \mathrm{MPa}$ at $77 \mathrm{~K}$.

The twinning CRSS of CoCrNi calculated by Huang et al. ${ }^{[41]}$ was $291 \mathrm{MPa}$. Therefore, the dislocation slip was easier to be activated at the onset of yielding and at low levels of deformation. However, the temperature dependence of the twinning CRSS was observed to be almost negligible. Twin nucleation occurs once the stress reached the twinning CRSS. Therefore, twins were more likely to nucleate at CTs due to the higher slip stress close to the corresponding twinning stress. As widely acknowledged, the twin boundaries play dual roles, namely, increasing the strain hardening rate, as well as promoting uniform deformation and delaying the occurrence of necking. This is one of the important reasons for the excellent performance of this alloy at CTs.

Huang et al. ${ }^{[41]}$ proposed that a strong correlation exists between the twinning CRSS and the mechanical strength and ductility in CoCrNi-based MEAs. The calculated twinning CRSSs of CoCrNi, CoCrFeNi, CoCrMnNi and CoCrFeMnNi were found to be 291, 274, 277 and $236 \mathrm{MPa}$, respectively. It was observed that a larger CRSS corresponded to a higher strength and ductility. 

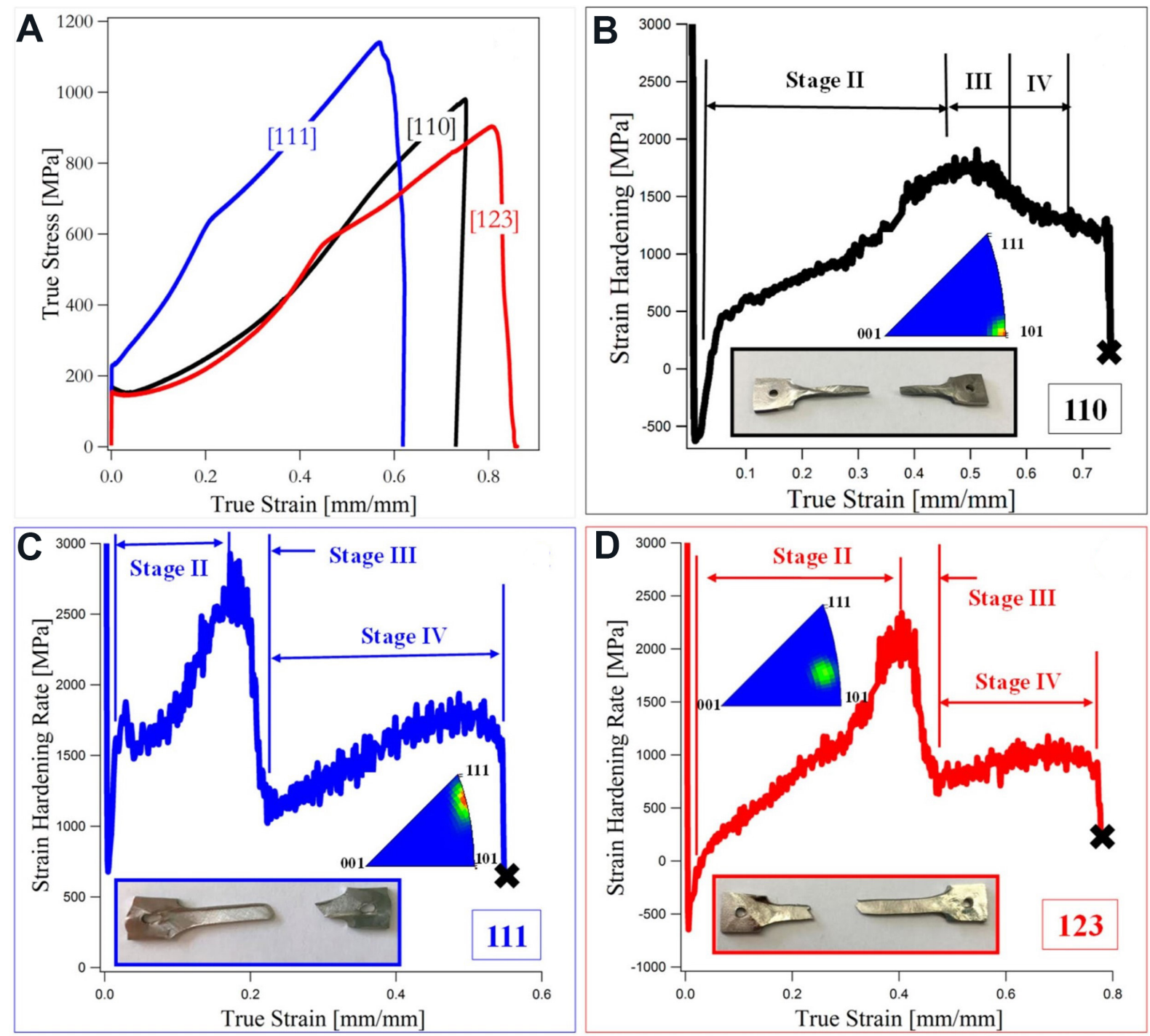

Figure 5. (A) True stress-strain response in CoCrNi single crystal samples oriented along the [110], [111] and [123] orientations deformed to failure under uniaxial tensile loading. Evolution of strain hardening rate as a function of true strain in the (B) [110], (C) [111] and (D) [123] orientations ${ }^{[66]}$.

\section{Stacking fault energy}

The SFE is defined as the energy per fault area by dissociating a perfect dislocation into the Shockley partial dislocations and is considered as a surface tension pulling the partials. It is one of the most important parameters to determine the deformation microstructural schemes among the slip, twinning and/or phase transformation. It is affected by the temperature and chemical composition:

(1) Temperature: In the case of the CoCrNi alloy, the experimental measurement of SFE at RT yielded a value of $22 \mathrm{~mJ} \cdot \mathrm{m}^{-2[32]}$. Many density functional theory calculations have shown that the SFE of the alloy at $0 \mathrm{~K}$ is negative ${ }^{[29,68,69]}$ and it decreases with decreasing temperature. A low SFE is conducive to the formation of twins and phase transformation, thereby enhancing the properties of the alloy. 
(2) Chemical composition: In a previous study, CoCrNi and CoCrFeMnNi were processed similarly and exhibited nearly random textures and almost the same grain sizes ${ }^{[32]}$. It was found that the separation between the Shockley partials in $\mathrm{CoCrNi}$ was wider than in $\mathrm{CoCrFeMnNi}$. This proved that the former had a lower SFE. Due to the lower SFE, nano-twinning was activated in $\mathrm{CoCrNi}$ at true strain between $4.0 \%$ and $6.7 \%$ at $77 \mathrm{~K}$ and between $9.7 \%$ and $12.9 \%$ at $293 \mathrm{~K}$, which was earlier than that of the CoCrFeMnNi alloy. Thus, the CoCrNi alloy exhibited superior properties than that of the CoCrFeMnNi alloy, which can be attributed to the lower SFE. It made the nano-twinning appear earlier and played a dominant role in a larger range.

Zhang et al..$^{[70]}$ used first-principles calculations to analyze the changes in the SFE by varying the content of $\mathrm{Co}$ and $\mathrm{Cr}$ in the CoCrFeMnNi alloy. It was observed that the SFE decreased with an increase in the contents of Co and Cr. In the CoCrNi alloy, there have been a few reports regarding changing the SFE by changing the alloy composition ${ }^{[46,71,72]}$.

Deng et al.$^{[46]}$ developed $\mathrm{Co}_{\mathrm{x}}(\mathrm{CrNi})_{(100-\mathrm{x})}[\mathrm{x}=33(\mathrm{Co33}), 40(\mathrm{Co} 40)$ or $50(\mathrm{Co50})$ at.\%] alloys and found that as the Co content increased, the proportion of twin boundaries increased and the average grain size decreased. The study proved that Co effectively reduced the SFE of the CoCrNi alloy.

Subsequently, $\mathrm{CoCr}_{x} \mathrm{Ni}$ alloys have also been systematically investigated ${ }^{[72]}$. The strength and ductility of the alloys were improved simultaneously with an increase in the $\mathrm{Cr}$ content. Compared with the CoNi alloy, the CoCrNi MEA offered a lower SFE. Deformation twins were significantly thinner. The dislocation pattern was transformed from the dislocation cells to slip bands. The Co-Cr-Ni ternary phase diagram ${ }^{[22,23]}$ suggests that the $\sigma$ phase precipitated with increasing $\mathrm{Cr}$ content. Thus, the effect of adding $\mathrm{Cr}$ on the SFE could be more complicated.

\section{Short-range order}

Tailoring the local chemical order affects defects, including vacancies, interstitials, twin boundaries and stacking faults, and therefore influences the macroscopic mechanical properties of an alloy ${ }^{[37]}$. These defects not only affect the strength, ductility and toughness but also modulate the resistance to high-temperature creep and even irradiation damage ${ }^{[31,73,74]}$. It is therefore essential to consider the effect of local chemical order in studies of the structure-property relationships of HEAs.

The Cr in the CoCrNi alloy tends to bond with $\mathrm{Ni}$ and Co and form SRO. This SRO can contribute to low electrical and thermal conductivities ${ }^{[33]}$. Increasing the amounts of such order leads to an increase in the SFE and hardness. Moreover, high-temperature aging can lead to the formation of appreciable SRO in the CoCrNi alloy ${ }^{[75]}$.

Ding et al.$^{[37]}$ studied the nature of local chemical order and established a relationship with SFE. The average SFE of the CoCrNi MEA varied remarkably with an increase in the chemical SRO [Figure 6]. They revealed four important characteristics of SFE in the CoCrNi alloy from calculations and analysis:

(1) The average SFE was highly tunable by tailoring the local chemical ordering;

(2) The low SFE of the CoCrNi MEA promoted the formation of deformation twins;

(3) The tunable SFE affected the deformation-induced phase transformation from FCC to the hexagonalclosed-packed (HCP) phase;

(4) The SFE exhibited a large distribution in values that would be realized in an alloy, where the state of the local chemical order varies heterogeneously throughout the sample. 

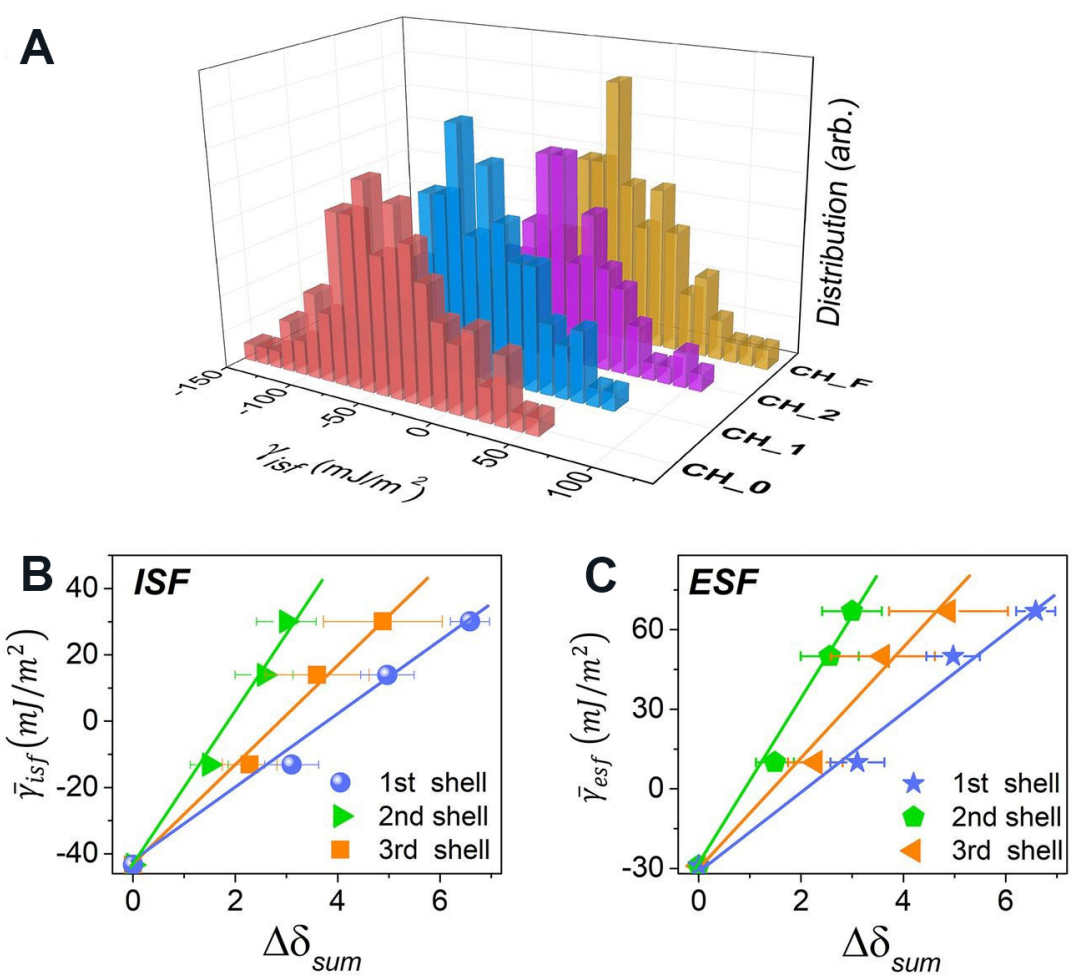

Figure 6. SFE correlates strongly with local chemical ordering. (A) Distribution of intrinsic SFE, $\gamma_{\text {isf }}$ for the CoCrNi alloy in four specific states, which span from a random solid solution to the highest degree of chemical ordering extracted from Monte Carlo simulations. Average SFEs, $(B) \bar{\gamma}_{\text {isf }}$ and $(C) \bar{\gamma}_{\text {isf }}$ among the four groups are plotted vs. $\Delta \delta_{\text {sum }}$ for the first, second and third nearest neighbor shells ${ }^{[37]}$. SFE: Stacking fault energy.

In contrast, Yin et al. ${ }^{[76]}$ showed that standard spin-polarized density functional theory calculations of misfit volumes were not accurate for the CoCrNi MEA. Thus, under typical processing conditions, the SRO in CoCrNi MEA was either negligible or had no systematic measurable effect on the strength.

As it is difficult to measure and control the SRO and there is no mature theory to prove its strengthening effect, more related research needs to be carried out in this area.

\section{Lattice distortion}

Jian et al. ${ }^{[34]}$ investigated the role of LD and SRO in the nucleation and evolution of dislocations and nanotwins in single-crystal and nanocrystalline CoCrNi alloys. They showed that YSs were determined by the strain to nucleate the Shockley partial dislocations. Higher degrees of LD lowered this strain, while a higher level of SRO increased it. After yielding, due to the increased sliding resistance caused by LD and SRO, nucleation of the nanotwins occurred during the reactions of mobile Shockley partials. Tong et al. ${ }^{[36]}$ proposed that the SRO possibly caused a large LD in the CoCrNi alloy. Therefore, the influencing factors are not independent of each other.

\section{Heat treatment}

The most obvious effect of heat treatment on properties is hardening via annealing. Praveen et al. ${ }^{[77]}$ reported that the annealing-induced hardening in the $\mathrm{CoCrNi}$ alloy was due to the changes in dislocation density and grain boundary relaxation. The dislocation density decreased but the grain size did not change significantly, indicating that only recovery occurred. Since the dislocation source is not abundant in 
nanocrystalline metals, deformation needs to be driven by a higher stress. The other reason is attributed to the changes in the grain boundary structure. The generation of dislocation from the relaxation is more difficult, which results in an increased resistance to the plastic deformation.

Schuh et al..$^{[78]}$ annealed the sample after high-pressure torsion (HPT) at $500{ }^{\circ} \mathrm{C}$ for $200 \mathrm{~h}$ and found that a minority phase was embedded in the FCC matrix. The phase showed an HCP structure. A possible reason could be the favorable segregation of Co that stacked the faults and led to a decrease in the SFE.

\section{Preparation method}

(1) Additive manufacturing (AM): The alloys discussed above were as cast. In recent years, AM technology has been gradually used to prepare HEAs ${ }^{[3,79-81]}$. AM technology is more efficient and versatile and can directly prepare parts with complex structures. This preparation method is known to improve the strength of the alloys. The high YS of the as-built state alloy was derived from the combined effects of lattice friction stress, boundary strengthening and dislocation strengthening ${ }^{[79]}$. Interestingly, the SFE of the CoCrNi alloy prepared by AM was lower than the as-cast one ${ }^{[38]}$. Niu et al. ${ }^{[80]}$ studied the effect of process parameters on the alloy and found that the sample density exhibited a non-monotonic relationship with the volume energy density (VED). The density initially increased, followed by a subsequent decrease, while the input VED gradually increased. The mean grain size and residual stress increased with an increasing VED, which produced more microcracks and deteriorated the tensile mechanical properties of the samples.

(2) Powder metallurgy (PM): The PM process has been used for the densification of bulk alloys ${ }^{[82]}$. It reduces the internal defects of the alloys and improves their properties. On this basis, it has been proved that advanced composite materials can be produced using PM. It can be seen from Figure 7 that the YS of the alloys prepared by PM was greatly improved, exceeding that of the alloy prepared by AM.

Moravcik et al. ${ }^{[83]}$ explored the mechanical properties of the CoCrNi alloy produced by mechanical alloying with subsequent spark plasma sintering (SPS). The alloy consisted of a major FCC phase $(94.4 \%)$ and a minor fraction of the body-centered cubic (BCC) phase (5.6\%). This was contrary to the single-phase alloy produced by the casting process, which is likely due to the different cooling kinetics.

\section{Textural evolution}

Sathiaraj et al ${ }^{[84]}$ investigated the textural evolution of the CoCrNi MEA during cold rolling. Up to intermediate rolling reductions, an $\alpha$-fiber brass-type texture developed, which was characterized by the intensities between Goss and Brass and negligible intensities near $\mathrm{Cu}$ and $\mathrm{S}$. A further heavy deformation strengthened this texture.

Slone et al ${ }^{[43]}$ correlated the nanoscale deformation mechanisms with the textural development. Almost all initial orientations rotated in the same direction to develop a strong $\langle 111>$ texture along the tensile axis, while the $<001>$-oriented grains remained stable. In contrast, a significant monotonical decrease of the $<110>$ fiber was observed [Figure 8]. The role of deformation twins in the development of texture could be ignored as it was mainly driven by slip instead.

The effect of annealing on textures was also studied ${ }^{[85-87]}$. After annealing, the $\alpha$-fiber textures were retained. The texture strength increased with increasing annealing temperature. The annealing temperature had little effect on the volume fraction of different texture components ${ }^{[85]}$. Although new orientations, BR, K, M and $\mathrm{D}$, emerged through annealing the twins, the overall texture intensity was weak after annealing ${ }^{[8,87]}$. 


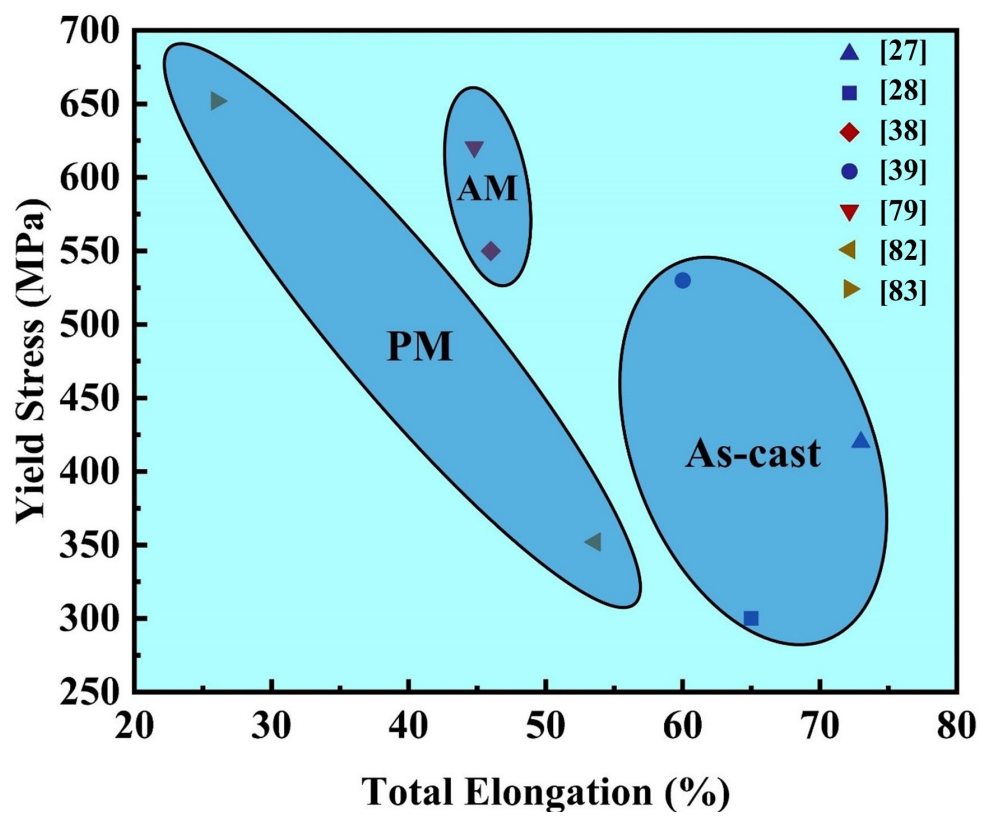

Figure 7. Comparison of tensile response of alloys prepared by different preparation methods ${ }^{[27,28,38,39,79,82,83]}$.

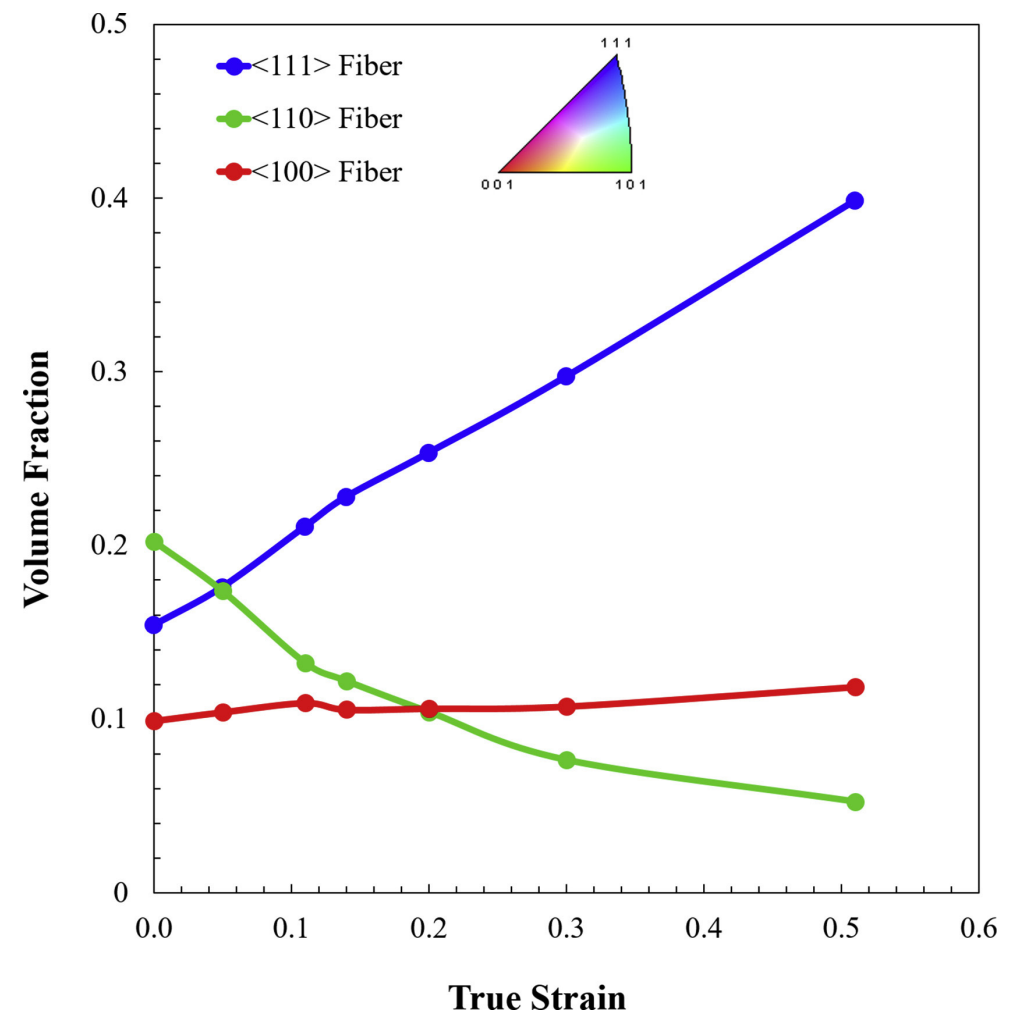

Figure 8. Evolution of volume fraction of different texture components as a function of true strain ${ }^{[43]}$.

\section{DEFORMATION MECHANISMS}

The deformation mechanisms of the CoCrNi alloy have been extensively studied. Through various characterization methods, the mechanisms at RT and CTs have been deciphered. However, the deformation 
mechanism at high temperature has scarcely been reported.

\section{Deformation mechanism at RT}

The deformation at RT can be approximately divided into three stages, namely, the dislocation slip, deformation twin and phase transformation stages. In addition, some other structures appear in the deformation process, such as stacking faults and nanotwin-HCP lamellae. Recently, dual-phase transformations have also been found under large strain.

\section{Dislocation slip stage}

The initial stage plasticity is characterized by the glide of $1 / 2<110>$ dislocations dissociated into two $1 / 6<112>$ Shockley partials on $\{111\}$ planes with a stacking fault in between. The separation between the Shockley partials ranges from $\sim 5 \mathrm{~nm}$ near the screw to $\sim 11 \mathrm{~nm}$ near the edge ${ }^{[32]}$. These relatively wide dissociations hamper the cross-slip and promote the planar slip.

Due to the occurrence of a large number of stacking faults, the interaction between them is very frequent. Networks of extended and contracted nodes were observed in the CoCrNi alloy [Figure 9A]. Figure 9B shows the presence of the extended stacking faults on different $\{111\}$ planes that intersect each other. Stacking faults that are sheared during the deformation typically leave behind interfacial partial dislocations ${ }^{[88]}$. These interfacial dislocations can be mobile and their glide may lead to the thickening or thinning of stacking faults. Further interactions may lead to two intrinsic stacking faults forming on successive $\{111\}$ planes to first produce an extrinsic stacking fault and finally producing nanotwins when three or more intrinsic stacking faults form on successive $\{111\}$ planes.

\section{Deformation twin stage}

As the deformation progresses, the stress reaches the twin CRSS and the nanotwins are activated. Twinning promotes a high work hardening rate by introducing extra boundaries that act as barriers to the dislocation motion (dynamic Hall-Petch effect), which postpones the onset of necking and increases the ductility.

Through in-situ transmission electron microscopy (TEM), Zhang et al. ${ }^{[29]}$ found that a three-dimensional (3D) hierarchical twin network is formed within individual grains [Figure 10]. Remarkably, while the twin boundaries contribute to the strengthening by acting as a barrier to the dislocation motion, the interconnected twin boundaries in the $3 \mathrm{D}$ twin network can also generate significant ductility by offering multiple pathways for dislocation motion along the twin boundaries.

\section{Phase transformation stage}

At a higher deformation value, nanotwin-HCP lamellae form and become a predominant aspect of the substructure. The HCP laths formed are usually a few atomic layers thick and are located next to the FCC deformation twins ${ }^{[28]}$. Annular dark-field scanning TEM (ADF-STEM) was used to reveal the detailed features of the boundaries of the subgrain structures formed at larger plastic strains. Figure 11 shows the deformation substructure in the specimen tested at $25 \%$ true strain at RT. Figure $11 \mathrm{~B}$ shows that HCP laths form, interspersed with the nanotwins, resulting in the nanotwin-HCP lamella. The HCP laths have the same orientation relationship with the matrix grain, with $(0001)_{\mathrm{HCP}} \|(111)_{\mathrm{FCC}}$ and $[11-20]_{\mathrm{HCP}} \|[1-10]$ characteristically, share the same $\{111\}$ habit planes as the coherent boundaries of the nanotwins.

The high-angle ADF-STEM (HAADF-STEM) image in Figure 12A shows a complex nanotwin-HCP lamella in a specimen strained to 53\% strain at RT. There are both well-developed multilayer HCP structures and local HCP stacking due to the slip of the single partial dislocations at twin boundaries 


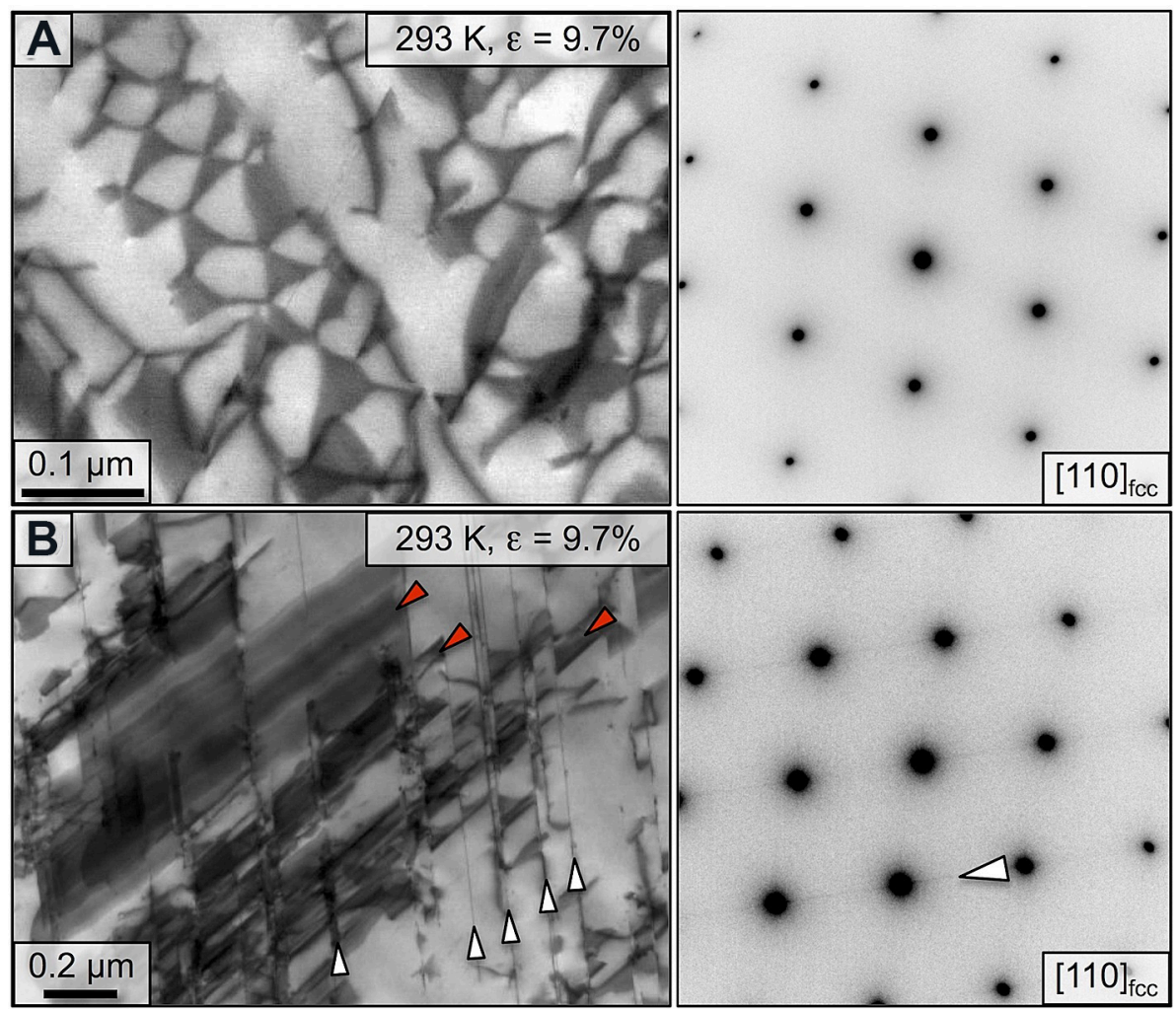

Figure 9. Microstructural features observed prior to mechanical twinning. (A) Network of in-plane dislocations showing extended nodes and corresponding diffraction pattern. (B) Interactions of two families of stacking faults, where the stacking faults of the first family are edge-on (indicated by white arrowheads) and the stacking faults of the second family are close to in-plane (indicated by red arrowheads). The corresponding diffraction pattern shows streaks perpendicular to edge-on stacking faults ${ }^{[32]}$.

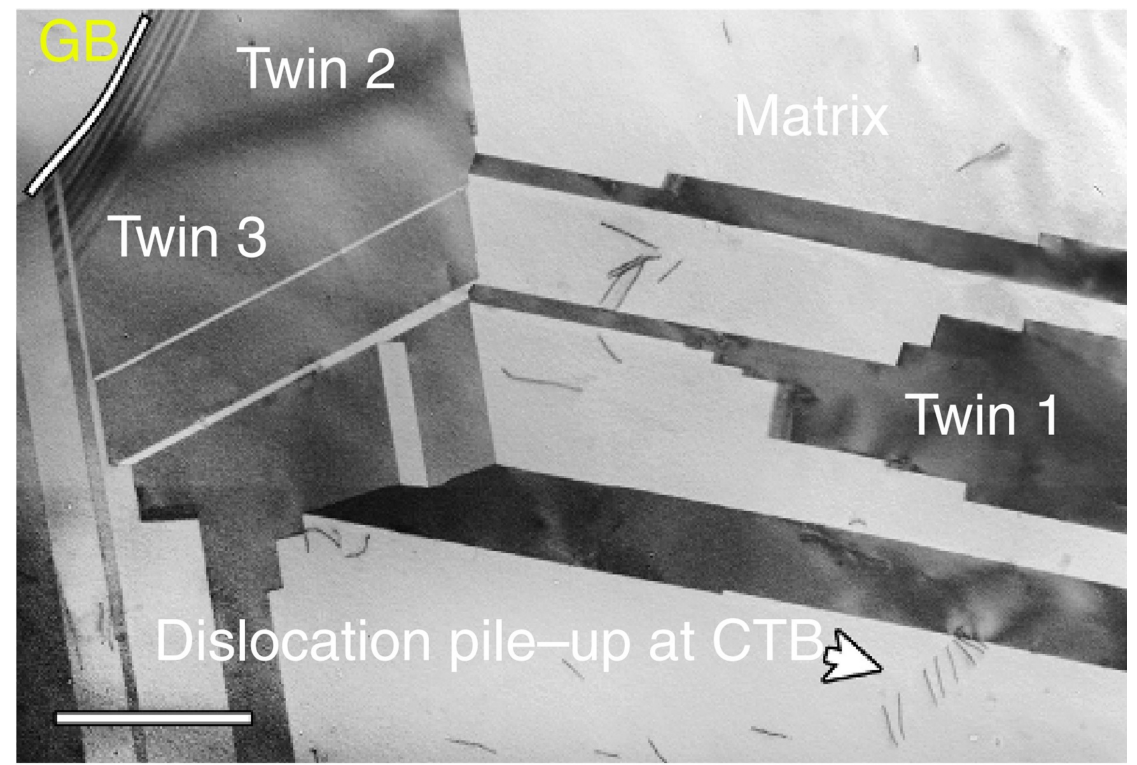

Figure 10. Bright-field TEM image showing the hierarchical twinning architecture in a grain of $\mathrm{CoCrNi}$. A grain boundary is marked by the white line near the top-left corner and the multiple twinning systems are labelled. Scale bar of $1 \mu \mathrm{m}^{[29]}$. 

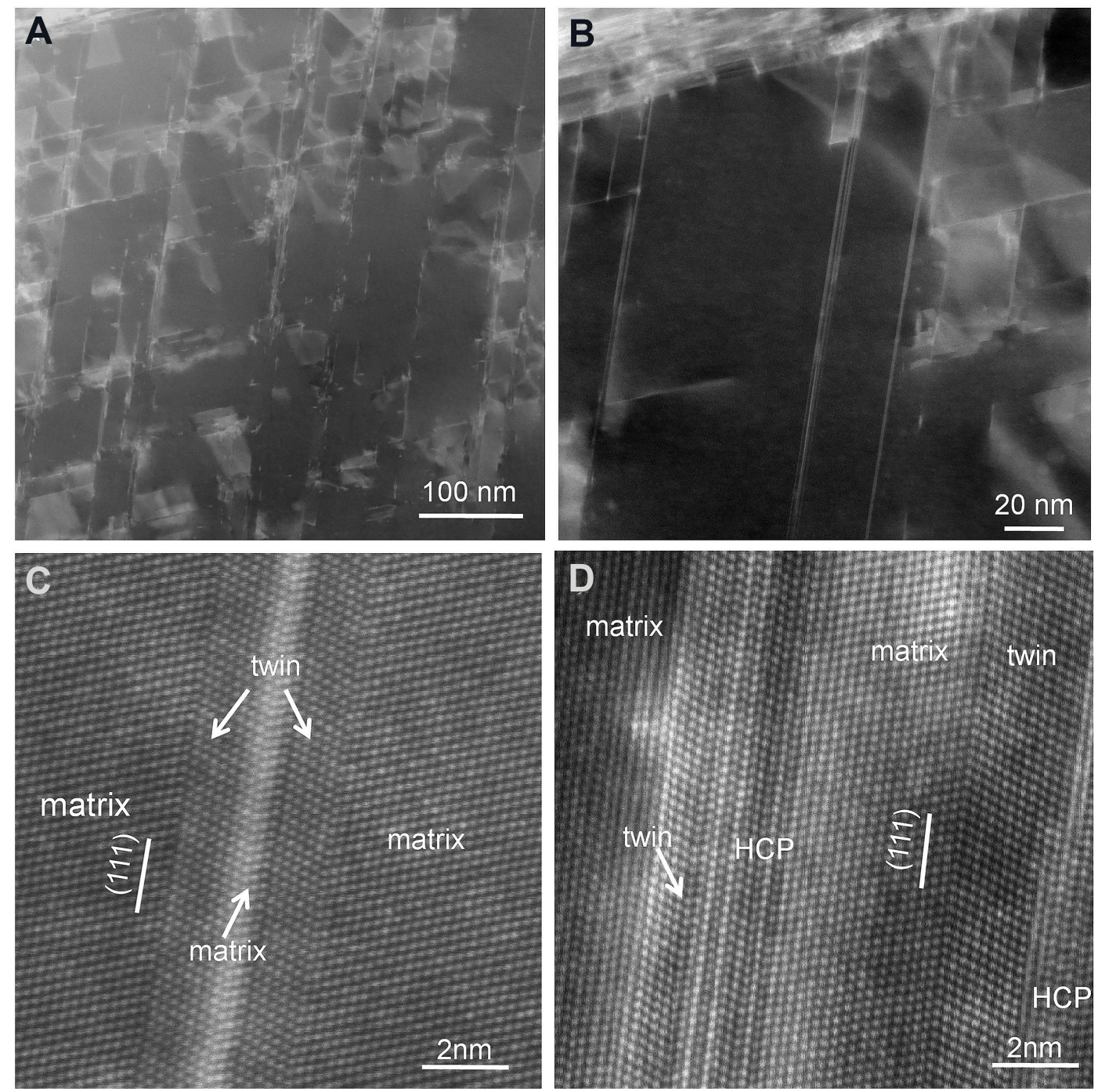

Figure 11. ADF-STEM imaging along a $<110>$ zone of a deformation substructure in the CoCrNi alloy after RT testing to a strain level of $25 \%$. (A) Formation of subgrain deformation substructure. (B) Details of subgrain deformation structure. (C) Nanotwin lamellae. (D) Formation of narrow HCP structure within a nanotwin lamella ${ }^{[28]}$.

[Figure 12B]. Thus, phase transformations could refine the grains and maintain large lattice rotations.

Atomic simulations by Niu et al.$^{[69]}$ showed that mixed and edge dislocations were unable to penetrate the nanotwin-HCP lamellae and instead forced to shear along with those features. This distributed the stress concentrations and promoted large hardening rates and ductility.

Recently, Chen et al. ${ }^{[4]}$ found dual-phase transformations for a CoCrNi film. The dual-phase transformations stem from a unique capability of facile slip on the $\{111\}_{\mathrm{FCC}} \|(0001)_{\mathrm{HCP}}$ planes in both the FCC and HCP phases of this alloy. Such dual-phase transformations can produce the intermediate HCP and FCC structures and their mixtures, enabling the facile plastic deformation and high ductility of the alloy. Dualphase transformation was previously not observed in the bulk materials, which may be due to the lack of a detailed atomistic characterization of the structural evolution sequence or a lack of high stresses to overcome the energy barriers for the dual-phase transformation. 

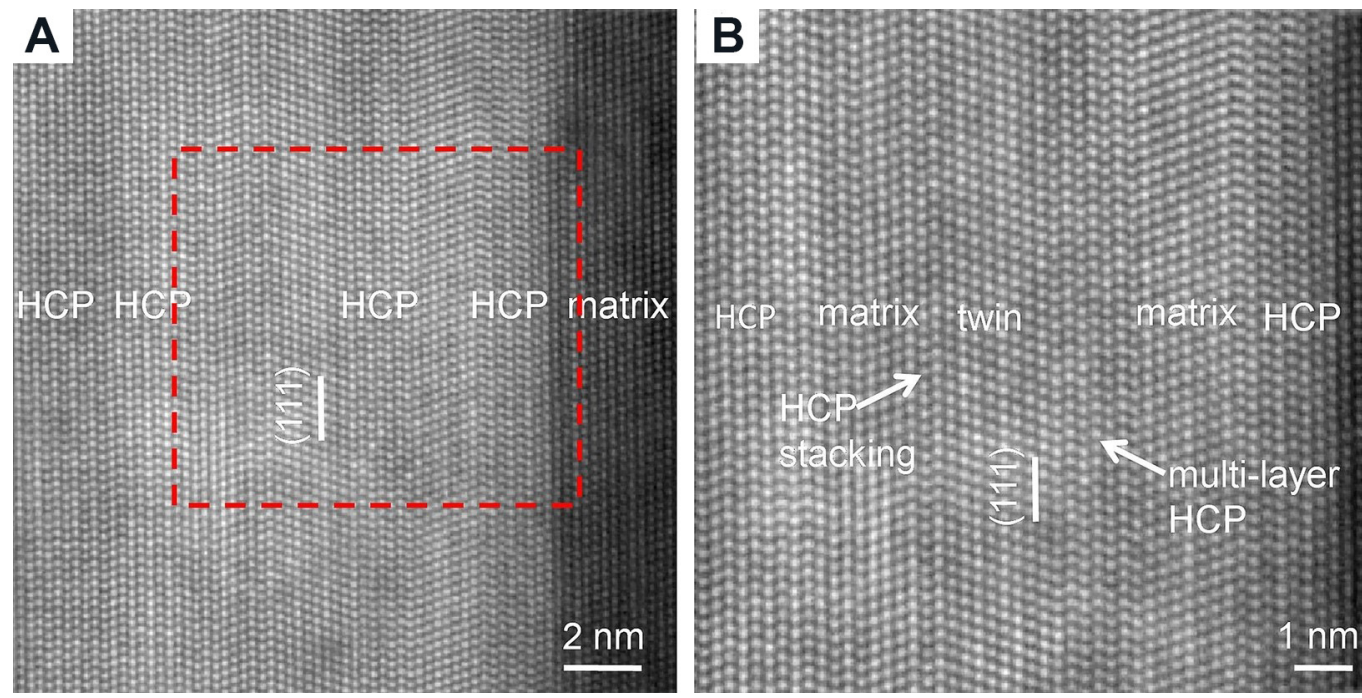

Figure 12. HAADF-STEM images of CoCrNi alloy tested to 53\% true strain at RT. (A) HAADF-STEM image of a well-developed parallel nanotwin-HCP lath structure. (B) A higher magnification HAADF-STEM image showing the highlighted region in $(A)^{[28]}$. HAADF-STEM: High-angle annular dark-field scanning transmission electron microscopy; HCP: hexagonal-closed-packed.

\section{Deformation mechanism at CTs}

The deformation mechanism at CTs is similar to the deformation mechanism at RT and can be divided into three stages. However, the massive cross-slip of the screw dislocations are found at CTs. A significant dislocation interaction was observed at the intersection of two slip systems, where the local stress concentration led to the activation of the new slip systems. This high frequency and density of the cross-slip resulted in a homogeneously distributed micro-slip inside the grains and promoted strain hardening. The extensive cross-slip activities and the subsequent dislocation multiplication and interactions was found to be related to the high strength during plastic deformation of the CoCrNi alloy at CTs ${ }^{[8]}$. Another difference is that the volume fraction of the nanotwin-HCP lamella increased more rapidly with strain at CTs than at RT. This phenomenon explains the high strain hardening rate at CTs.

Furthermore, the effect of HCP nano-lamellae on the tensile response in the CoCrNi alloy was investigated by employing molecular dynamics simulations ${ }^{[45]}$. Ma et al ${ }^{[45]}$ found that the overall strengthening had two aspects: phase strengthening and extra interface strengthening. The interface strengthening was always stronger than the phase strengthening. The overall and interface strengthening increased with an increase in the lamellar width and a decrease in the interspacing. Because of the increase of lamellar width, the blocking ability of the dislocation slip was stronger and the density of the phase boundaries was higher when the interspacing was smaller, thus providing more barriers for the dislocation glid in the other slip systems and resulting in higher strength.

\section{STRENGTHENING METHODS}

Like other metal materials, the most promising applications of HEAs are considered to be in the structural field. Therefore, abundant efforts have been focused on the study of their mechanical properties, particularly strength and ductility. It is well known that the mechanical properties of crystalline materials can be improved through modifying microstructures (such as grain size, dislocations and twins) and/or chemical structures (such as alloying). The optimization of microstructures can be realized by controlling the process of deformation and heat treatment. Modifying chemical structures can introduce elements with large atomic radii to produce precipitation or interstitial atoms to increase the lattice distortion. These 
factors are not independent of each other. In fact, they are often interconnected. For example, the chemical and processing route directly affects the microstructure of the alloy.

\section{Optimizing microstructures}

Defects

Huang et al. ${ }^{[00]}$ tuned the formation of multiple lattice defects via SPS to strengthen the CoCrNi alloy. The as-SPS sample contains a high density of dislocation cells, small stacking faults, ultrafine nanotwins and Lomer-Cottrell locks.

Using torsion deformation, not only can the high dislocation density be produced, but also the gradient dislocation density. The high dislocation density and the high nanotwin volume fraction contribute to the high strength at the edge of the MEA bar. The low dislocation density and low nanotwin volume fraction lead to a good ductility in the center of the MEA bar. High strength and good ductility can be simultaneously achieved at a suitable gradient in the microstructure ${ }^{[91]}$.

In alloys with low SFE, the twins and HCP structures can also be designed for strengthening. The synergistic effect of the HCP lamellae structure and twins, including the nanotwins induced by an equal channel angular pressing at RT and the annealing twins induced by the post-deformation annealing at different temperatures, has been employed to obtain the desired mechanical properties ${ }^{[92]}$.

\section{Grain refinement}

Severe plastic deformation, followed by annealing, is an effective method to produce a microstructure with an ultrafine grain. The most common deformation method is rolling. However, HPT is needed to obtain more severe deformation, owing to its application of high hydrostatic compressive stress, thereby achieving high strain in the material without undesirable cracking.

Praveen et al. ${ }^{[48]}$ prepared a nanocrystalline CoCrNi alloy with a grain size of $\sim 50 \mathrm{~nm}$. Its UTS and TE were $\sim 2.2 \mathrm{GPa}$ and $\sim 9 \%$, respectively. Moreover, ultrafine-grained CoCrNi was fabricated by HPT, followed by annealing ${ }^{[49]}$. The alloy exhibited superior cryogenic properties with a YS of $\sim 2 \mathrm{GPa}$ and a tensile strain of $\sim 27 \%$. It was found that the cryogenic YS of the ultrafine-grained sample increased by $67 \%$ compared to that of the coarse-grained sample.

\section{Heterogeneous structure}

Alloys with coarse grains exhibit insufficient strength, which is not conducive to their application in structural engineering. Refining the grain size to the nanoscale can elevate the strength at the expense of ductility. Recent research shows that tailoring the microstructures of alloys can optimize their mechanical performance.

Overcoming the strength-ductility trade-off in various metals and alloys, including bimodal ${ }^{[93-96]}$, heterogeneous lamella [Figure 13 $]^{[97]}$ and gradient structures ${ }^{[88-103]}$, has been extensively studied. There is a dramatic difference in the strength between the different domains in these materials. The microstructures show significant heterogeneities. Thus, these materials can be considered as heterogeneous materials. Yoshida et al. ${ }^{[30]}$ demonstrated that the heterogeneous structure exhibited a better uniform elongation than the homogeneous structure. This strategy of alloy design has been utilized in HEAs to realize a superior combination of strength and ductility. 

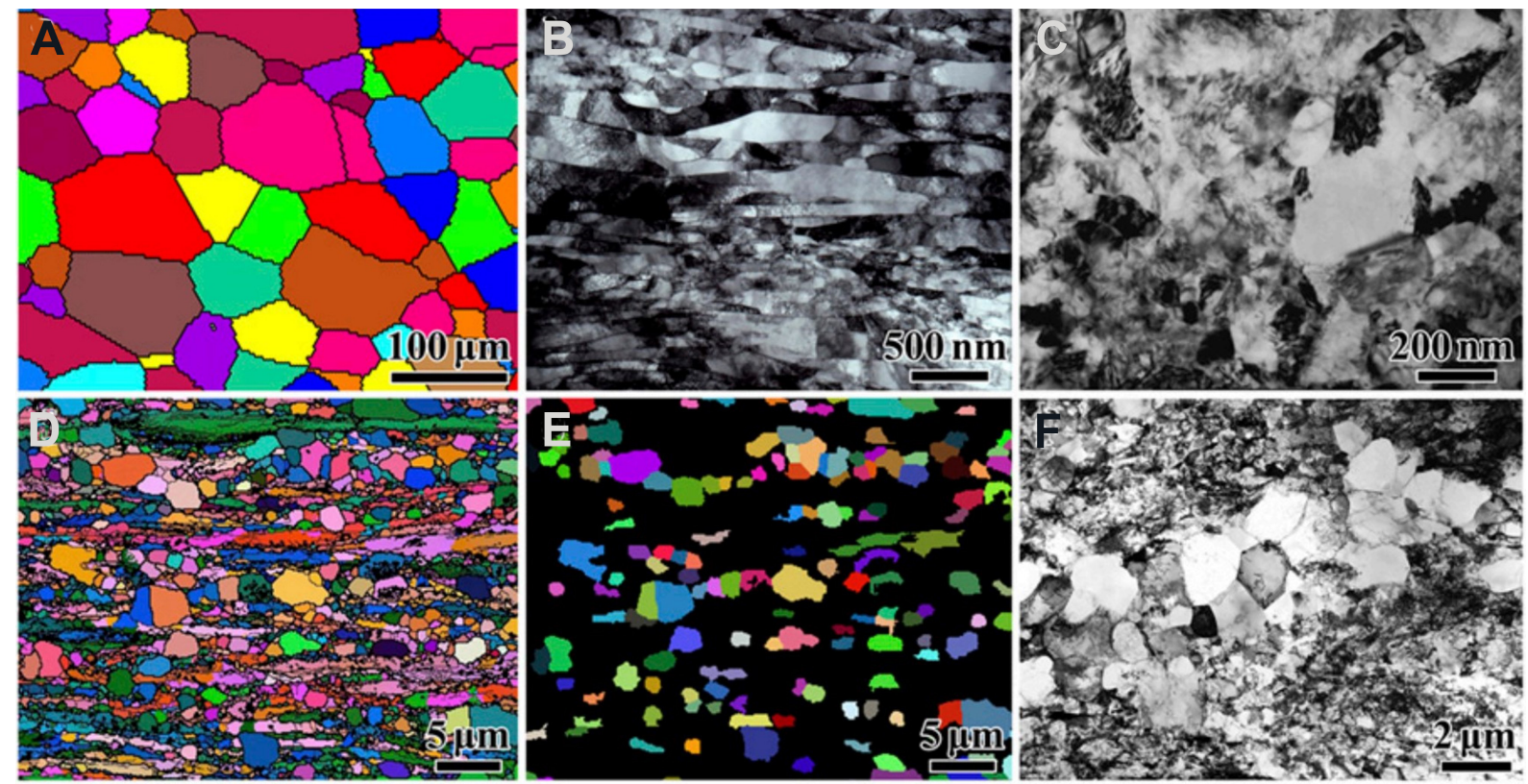

Figure 13. Microstructure of heterogeneous lamella Ti. (A) Electron backscattered diffraction (EBSD) image of initial coarse grain Ti. (B) and (C) TEM images showing nonuniform elongated lamellae in the central layer and nanograins in the top 25 $\mu \mathrm{m}$ surface layer. (D) EBSD image of heterogeneous lamella Ti after partial recrystallization. (E) EBSD image of recrystallized grains larger than $1 \mu \mathrm{m}$. (F) Cross-sectional TEM image of recrystallized grain lamellae with two ultrafine-grained lamellae on two sides ${ }^{[97]}$.

In complex composition alloys, the formation of multiple phases can provide heterogeneity. Multiple strengthening mechanisms (such as precipitation and twinning- and transformation-induced plasticity effects) operate simultaneously. However, single-phase FCC HEAs/MEAs can be subjected to the heterogeneity of multilevel grain structures to exhibit the properties achievable in multiphase heterogeneous alloys $^{[104]}$. The difference in the deformation behavior of recrystallized coarse, recrystallized fine and nonrecrystallized grains can cause strain incompatibility. To accommodate the strain incompatibility between the soft and hard regions, geometrically necessary dislocations (GNDs) need to be generated. The dislocation movement becomes difficult because of the long-range stress caused by GNDs, resulting in the strengthening of the back stress. Back stress hardening has almost no contribution to the YS in single-phase homogeneous grains. However, it could be obvious in the heterogeneous grain structure in single-phase alloys with low SFE. Heterogeneous structures are usually obtained by annealing the heavily deformed alloys at low temperatures for a short time ${ }^{[105-108]}$.

The deformation process in heterogenous materials can be divided into three stages, as shown in Figure 14. In stage I, both the soft and hard domains undergo elastic deformation. In stage II, the soft domains start to deform plastically, while the hard domains remain elastic. The soft domains cannot deform freely and need to deform compatibly with the neighboring hard domains. The plastic strain gradient can be generated due to the continuity of the strain at the domain boundaries. The strain gradient is accommodated by the GND. The soft domains can be enhanced, leading to an increase in the YS. In stage III, both the soft and hard domains deform plastically. However, the soft domains sustain much higher strain than the hard domains, which produces strain partitioning. As the strain partitioning increases, the back-stress strengthening plays a critical role in the deformation process, which prevents necking. Thus, the strength of heterogeneous materials can be increased without a serious loss of ductility ${ }^{[109]}$. 


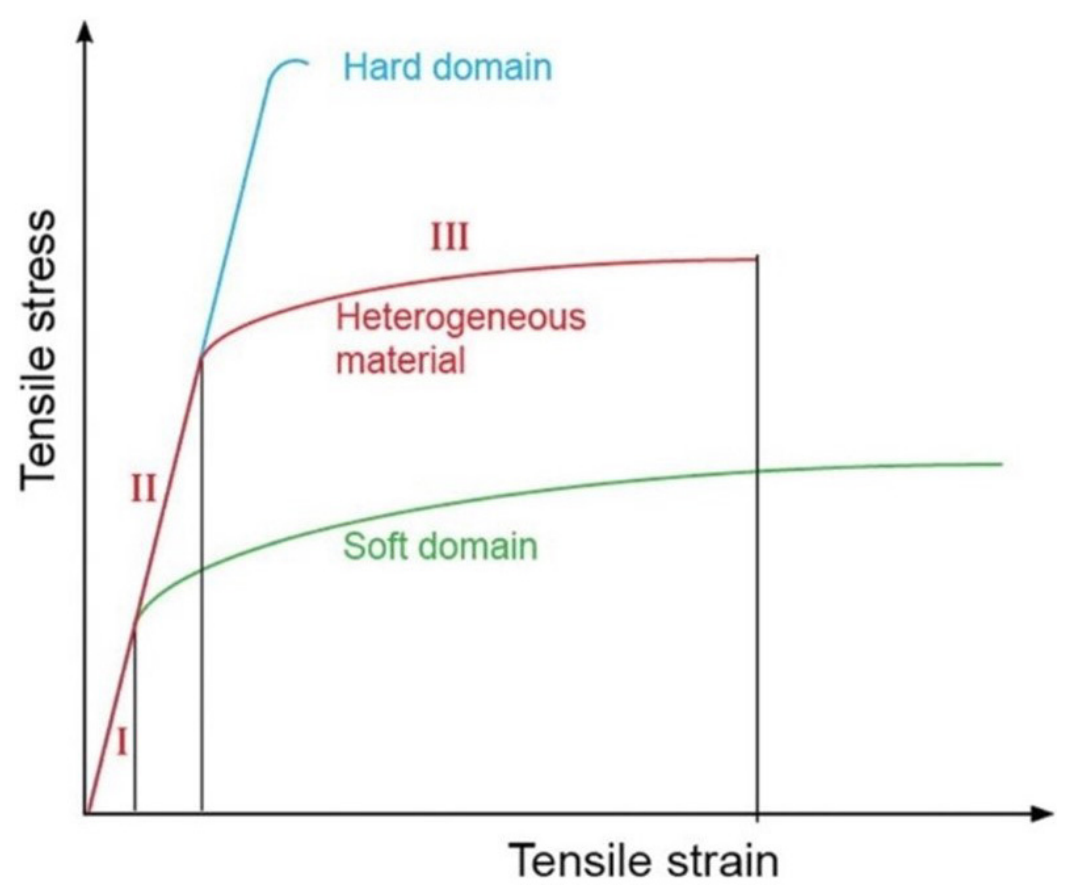

Figure 14. Three deformation stages of heterogeneous materials ${ }^{[109]}$.

The formation mechanism of the heterostructure in the CoCrNi MEA using TEM has been studied ${ }^{[10]}$. Due to low SFE, numerous twins nucleated at the corner of the high-angle grain boundaries to produce nanograins. Short-time partial-recrystallized annealing and abundant thermal twinning then facilitated the heterogeneous structure with multiple grain sizes.

Through molecular dynamics simulations, Yuan et al. ${ }^{[51]}$ investigated the atomistic deformation mechanisms of tensile deformation in the CoCrNi MEA with heterogeneous grain structures. As described above, a high YS and large ductility can be achieved by strain partitioning and back stress developed during the yielding and subsequent plastic deformation. Interestingly, the strain partitioning is more obvious by increasing the grain size ratio between the coarse and fine grains. Wen et al ${ }^{[50]}$ proposed that the macroscopic mechanical response of the heterogeneous microstructures can be treated as a rule-of-mixture composite of the asrolled and fully recrystallized materials with a similar grain size.

Another typical heterogeneous structure is the gradient structure. To achieve the heterogeneous gradient structure, some researchers have adopted the ultrasonic surface rolling processing technique ${ }^{[50]}$. The whole range of the gradient structure can be divided into three parts, namely, the severely deformed top surface, mediate transition and coarse grain matrix areas. Large numbers of slip bands intersect in the top surface area. Below the top surface area, the mediate transition area has a typical heterogeneous contrast of dislocation structures. The deepest part is the coarse grain matrix area, which is characterized by the obvious annealing twins. Compared with the coarse grain sample, the CoCrNi MEA with a heterogeneous gradient structure shows a four-fold increase in the YS, together with a considerable tensile strain to failure of $\sim 58.7 \%$.

\section{Alloying}

Alloying elements have a first-order effect on strength and ductility ${ }^{[111]}$. In HEAs, adjusting one element may have profound effects on the mechanical properties. Researchers have optimized the properties by 
controlling the types and contents of different elements.

Substitutional elements ( $\mathrm{Al}, \mathrm{Fe}, \mathrm{Mo}, \mathrm{W}, \mathrm{Cu}, \mathrm{Nb}$ and $\mathrm{Ti}$ )

$\mathrm{Al}$ has a significant impact on the alloy properties due to its large radius. The microstructure evolves from FCC to FCC and BCC and then to double BCC structures with an increase in the Al content ${ }^{[12-115]}$. This is accompanied by a decrease in Young's modulus and Poisson's ratio and an increase in the tensile YS and hardness ${ }^{[16]}$.

$\mathrm{Hu}$ et al.$^{[52]}$ investigated the grain growth behavior of $\mathrm{CoCrNi}$ and $\mathrm{CoCrNi}-3 \mathrm{Al}$ alloys. The activation energies for the grain growth of CoCrNi and CoCrNi-3Al were found to be $\sim 251$ and $\sim 219 \mathrm{~kJ} / \mathrm{mol}$, respectively, indicating that the addition of $\mathrm{Al}$ accelerated the grain growth due to the lower self-diffusion activation energy of Al.

The $\mathrm{Al}_{0.3} \mathrm{CoCrNi}$ alloy can obtain a heterogeneous structure through simple thermomechanical processing ${ }^{[117]}$. This alloy exhibits a remarkable combination of YS ( 945 MPa), UTS ( 1230 MPa) and total elongation $(\sim 38 \%)$. This can be attributed to the synergetic mechanism of the grain refinement, precipitation strengthening, heterogenous structure and nanoscale deformation twins. The alloy also exhibits excellent CT mechanical properties ${ }^{[118]}$.

$\mathrm{Fe}$ is a widely used alloy element. Its atomic radius is similar to that of $\mathrm{Co}, \mathrm{Cr}$ and $\mathrm{Ni}$ with a difference $<$ $0.8 \%$. Tong et al. ${ }^{[36]}$ quantitatively analyzed the lattice distortion of $\mathrm{CoCrNi}$ and $\mathrm{CoCrFeNi}$ alloys. They found that the local LD in $\mathrm{CoCrFeNi}$ was much lower than that in the $\mathrm{CoCrNi}$ alloy ${ }^{[36]}$. The relatively large local LD in CoCrNi was possibly caused by the SRO. In contrast, the stacking fault energy of the CoCrFeNi alloy at RT was $32.5 \mathrm{~mJ} / \mathrm{m}^{2[119]}$, which was higher than that of the CoCrNi alloy $\left(22 \mathrm{~mJ} / \mathrm{m}^{2}\right)^{[32]}$. Based on the above two aspects, the strengthening effect of $\mathrm{Fe}$ was not obvious in the CoCrNi alloy.

The activation energy for the grain growth of the CoCrNi-3Mo alloy calculated by $\mathrm{Hu}$ et al. ${ }^{[52]}$ was $368 \mathrm{~kJ} / \mathrm{mol}$, which is higher than the value of $251 \mathrm{~kJ} / \mathrm{mol}$ obtained for CoCrNi. This shows that the addition of Mo retards the grain growth. Moreover, the SFE of the Mo-doped alloy at o K was equivalent to that of the CoCrNi alloy ${ }^{[120]}$. The $\sigma$ phase is precipitated after the Mo element becomes supersaturated ${ }^{[121-123]}$. Chang et al.$^{[123]}$ obtained a heterogeneous banded precipitation using compositional inhomogeneity, which improves the strength and ductility synchronously compared to the conventional structure with the $\sigma$ phase formed at the grain boundaries.

In addition to casting, a Mo-coated CoCrNi mixed powder was designed and synthesized by a coating method, followed by calcination and reduction processes. Although the strength was improved in this process, the ductility was sharply reduced ${ }^{[124]}$.

It was observed that the heterogeneity in the chemical distribution increases by doping with a trace amount of $\mathrm{W}^{[53]}$. Figure 15 shows the strain maps of the CoCrNi-3W and CoCrNi alloys. The atomic-scale lattice strain in $\mathrm{CoCrNi}-3 \mathrm{~W}$ was more severe. The tensile and compressive strain fields alternated, which resulted in atomic-scale lattice strain fluctuations. This produced a high density of pinning points to resist the dislocation glide.

Due to the addition of $\mathrm{W}$, the recrystallized behavior of the alloy was retarded. The activation energy of alloys increased dramatically, indicating slow grain coarsening kinetics. Thus, the grain refinement contributed to the YS to a significant extent with an increase in the $\mathrm{W}$ content ${ }^{[125,126]}$. 


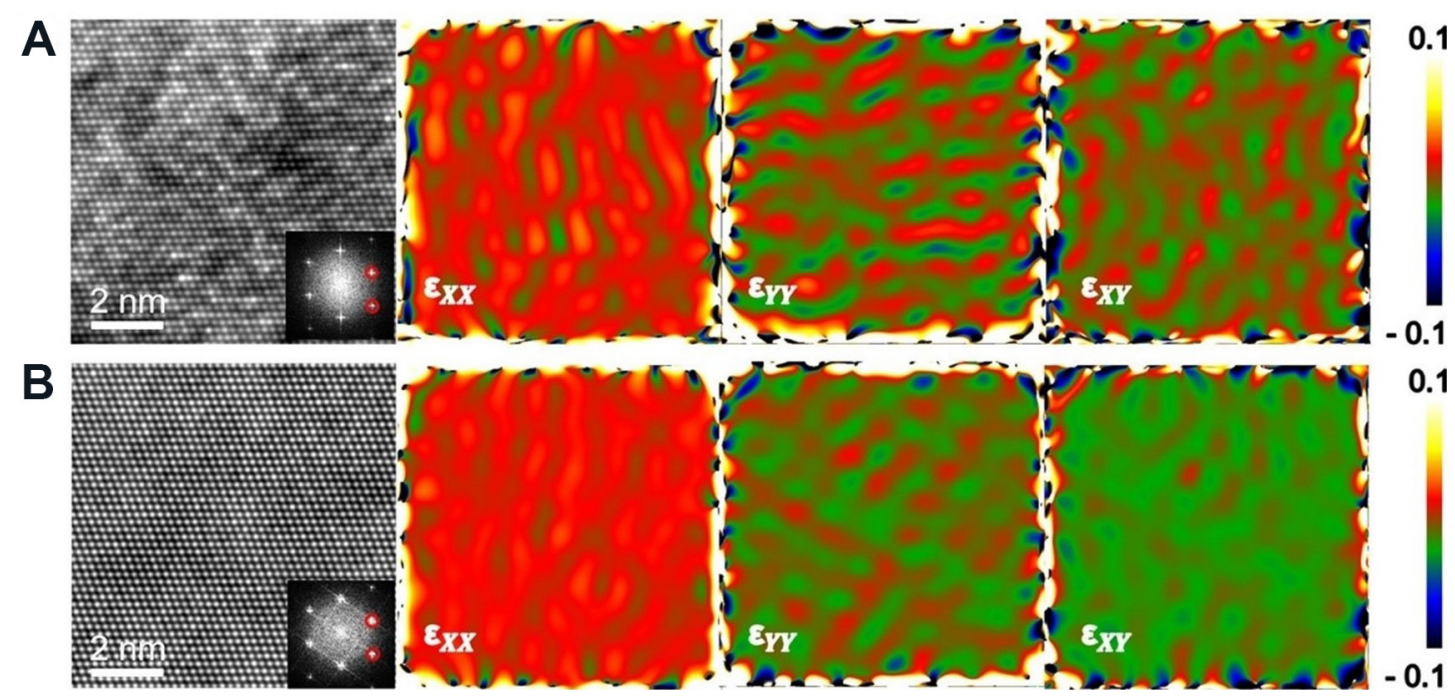

Figure 15. Comparison of atomic-scale strain distribution in (A) CoCrNi-3W and (B) CoCrNi ${ }^{[53]}$

The addition of $\mathrm{Cu}$ is also a common strengthening method but it does not usually become uniformly distributed in the matrix. Due to the tendency of the positive mixing enthalpy of $\mathrm{Cu}$ with other elements, the addition of $\mathrm{Cu}$ induces a phase separation phenomenon, causing the formation of a secondary phase.

The effects of $\mathrm{Cu}$ on the microstructure and recrystallization behavior have been investigated recently ${ }^{[54,27]}$. The $\mathrm{Cu}$ addition caused a phase separation, resulting in the formation of $\mathrm{Cu}$-lean FCC1 and $\mathrm{Cu}$-rich FCC2 phases. Compared with the $\mathrm{Cu}$-lean phase, the $\mathrm{Cu}$-rich phase exhibited a low hardness. This allowed most of the plastic deformation to be accommodated by the $\mathrm{Cu}$-rich phase. Moreover, the $\mathrm{Cu}$-rich phase tends to be extensively recrystallized than the $\mathrm{Cu}$-lean phase.

The addition of $\mathrm{Nb}$ into the CoCrNi matrix leads to the formation of a Laves phase with a HCP structure ${ }^{[5]}$. CoCrNiNbx alloys evolve from hypoeutectic $(0<\mathrm{x}<0.4)$ to eutectic $(\mathrm{x}=0.4)$ and then hypereutectic $(\mathrm{x}>$ 0.4 ) with an increment in the $\mathrm{Nb}$ content. An increase in the Laves phase causes the hardness and compression YS to increase and the compression fracture strain to decrease.

Ti is usually used to design low-density alloys. 3D nanolattices have recently emerged as an effective strategy to achieve high strength at low density. Feng et al. ${ }^{[56]}$ fabricated CoCrNiTi ${ }_{0.1}$ MEA-coated nanolattices that exhibit unprecedented surface wrinkling under compression.

\section{Interstitial elements ( $B, C, N$ and $\mathrm{Si}$ )}

Shi et al.$^{[57]}$ studied the micromechanical behavior of B doping in the CoCrNi alloy by in-situ high-energy Xray diffraction. It was found that $\mathrm{B}$ doping of the $\mathrm{CoCrNi}$ alloy resulted in an obvious partial recrystallization and significant refinement of the grain size.

A CoCrNi/B composite was prepared by mechanical alloying and SPS ${ }^{[128]}$. This processing method greatly refined the grains. The composite produced 11 vol.\% of a $\mathrm{Cr}_{5} \mathrm{~B}_{3}$ boride phase, thereby possessing high strength values, surpassing a UTS of $1400 \mathrm{MPa}$ and a tensile elongation of $1.86 \%$. 
Shang et al..$^{[58]}$ found that the addition of $\mathrm{C}$ increased the dislocation density of the alloy. The dislocation substructures were refined and the stress concentration caused by the planar slip was weakened. Meanwhile, $\mathrm{C}$ doping increased the SFE of the alloy, delayed the nucleation of the deformation twins and decreased the thickness of the twin bundles.

Due to the high affinity between $\mathrm{C}$ and $\mathrm{Cr}$, the alloys with high $\mathrm{C}$ contents could generate nanosized $\mathrm{M}_{23} \mathrm{C}_{6}$ carbides. $\mathrm{C}$ also retarded the recrystallization kinetics of the alloy by decorating the grain boundaries and hindering the interfacial motion through carbides that were formed during the recrystallization process. Therefore, solution, grain refinement and precipitation strengthening were found to play a simultaneous role in the CoCrNi alloy ${ }^{[129]}$.

In some steels, interstitial $\mathrm{N}$ doping was more advantageous than $\mathrm{C}$ doping due to its higher solubility and slower kinetics in the formation of nitrides compared to the formation of carbides ${ }^{[130-132]}$. $\mathrm{N}$ doping was found to be a cost-effective method to improve the mechanical properties of conventional alloys. Thus, the effect of $\mathrm{N}$ addition in HEA systems is certainly worthy of further study.

The effect of 0.5 at.\% interstitial $\mathrm{N}$ on the CoCrNi alloy was investigated in an earlier report ${ }^{[59]}$. The alloy exhibited a single-phase FCC lattice structure without nitrides. The addition of $\mathrm{N}$ increased the YS by $24 \%$ $33 \%$, while maintaining the same tensile ductility. Moravcik et al. ${ }^{[59]}$ attributed the strengthening effect to the increment in the elastic misfit and the associated dislocation interaction strength.

Some researchers have subjected the molten alloy to a $\mathrm{N}_{2}$ atmosphere for $\mathrm{N}$ addition ${ }^{[133,134]} \cdot \mathrm{N}_{2}$ gas was flowed for $24 \mathrm{~h}$ to saturate the alloy homogenously. The formation of $\mathrm{Cr}_{2} \mathrm{~N}$ precipitates results from the stronger affinity and higher solubility of $\mathrm{N}$ in $\mathrm{Cr}$ than in $\mathrm{Co}$ and $\mathrm{Ni}$. $\mathrm{The} \mathrm{Cr}_{2} \mathrm{~N}$ precipitates were observed to be semi-coherent with the FCC matrix. Thus, it hindered the grain growth via the Zener pinning pressure. It was also investigated that lower annealing temperatures with a large fraction of fine-sized $\mathrm{Cr}_{2} \mathrm{~N}$ resulted in a higher Zener pinning pressure ${ }^{[134]}$. Apart from improving the mechanical properties, the addition of $\mathrm{N}$ also improved the corrosion resistance of the alloy ${ }^{[135]}$.

$\mathrm{Si}$ is a lightweight and an inexpensive element. It can improve the mechanical properties of the alloys, such as the wear, corrosion and oxidation resistance, hardness and thermal stability. Unlike the addition of $\mathrm{C}$ and $\mathrm{N}$ elements, the addition of Si promoted the recrystallization rate and accelerated the growth of the grains on heating the alloy specimens. However, the specific reasons remain unknown. The thermodynamic model proves that the SFE in the alloys decreases with increasing Si addition. Thus, compared with the CoCrNi MEA, the Si-doped alloys exhibited a more concentrated and thinner distribution of deformation twins and multiple twinning structures [Figure 16] ${ }^{[60]}$. Both the strength and elongation of the alloys were found to be significantly improved with this strategy.

Yi et al ${ }^{[136]}$ found that as-cast $\mathrm{Si}_{0.3} \mathrm{CoCrNi}$ and $\mathrm{Si}_{0.4} \mathrm{CoCrNi}$ alloys exhibited dual FCC-phase structures. The Si-rich FCC phase located between the dendrites and increased the compression YS.

The addition of Si also improved the helium irradiation resistance of the alloy. The helium bubble growth tends to be delayed as Si was introduced ${ }^{[137]}$. It occurs via several interconnected mechanisms. Firstly, the high concentration of $\mathrm{Si}$ in the matrix accelerated the diffusion of vacancies, leading to lower vacancy supersaturation and delayed bubble growth. Secondly, the addition of Si enhanced the chemical disorder. It was found that increasing the compositional complexity and reducing the energy dissipation pathway results in the inhibition of the accumulation of point defects and reduces the damage caused by irradiation. 

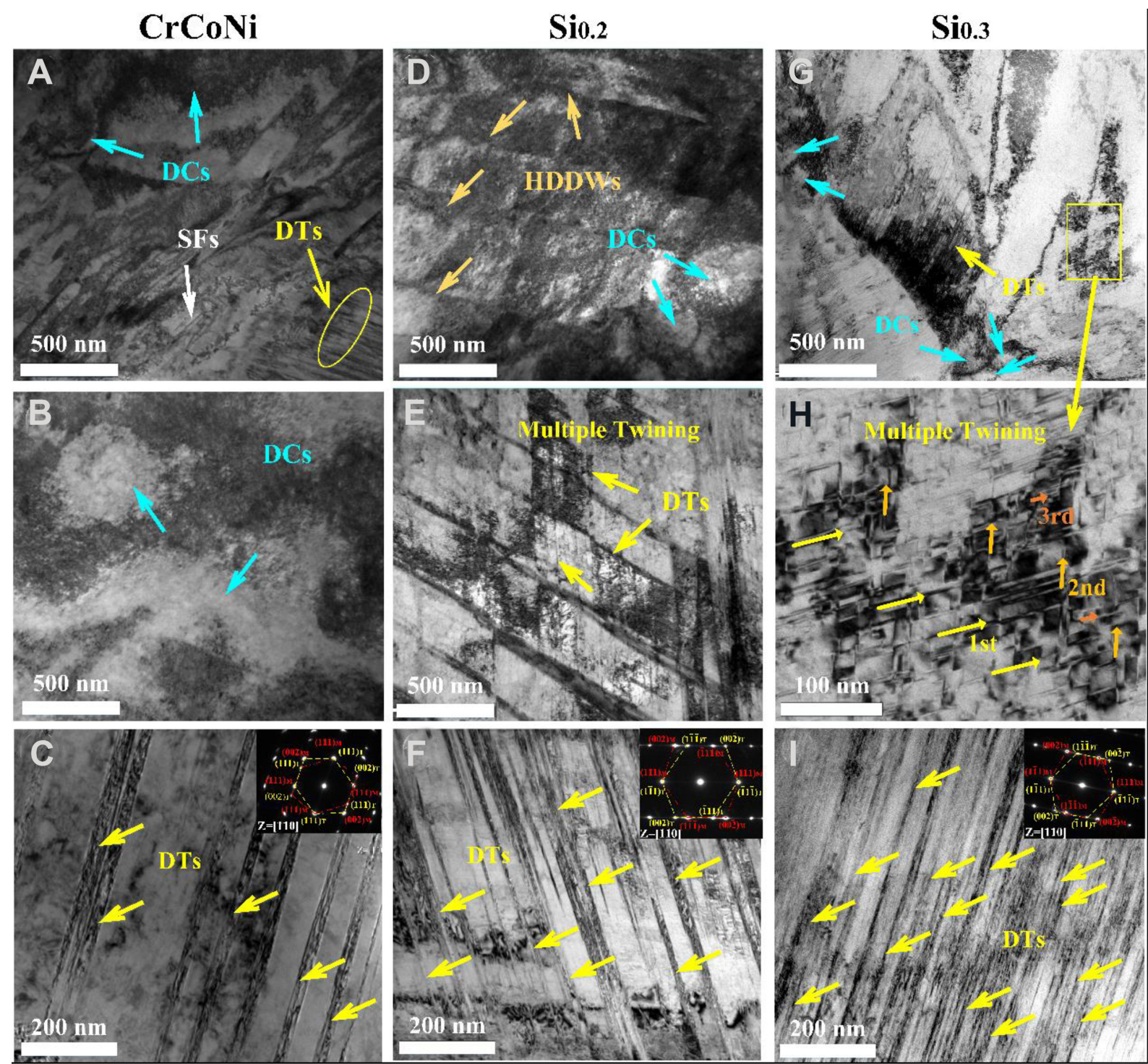

Figure 16. TEM micrographs showing the different characteristics of (A-C) $\mathrm{CoCrNi}_{\text {, }}\left(\mathrm{D}-\mathrm{F}\right.$ ) $\mathrm{CoCrNiSi}_{0.2}$ and (G-I) $\mathrm{CoCrNiSi}_{0.3} \mathrm{MEAs}$ at the fracture strain. (A), (B), (D) and (G) Bright-field images representing the main micromorphological characteristics and dislocation cells. (E) Bright-field images of multiple twinning in the alloys. $(\mathrm{H})$ Corresponding higher magnification image representing multiple twinning in the yellow rectangular area of (G). (C), (F) and (I) Bright-field images of twin bundles with their corresponding selected area electron diffraction patterns inserted ${ }^{[60]}$.

Thirdly, the lattice distortion caused by the introduction of Si atoms also played an important role in the helium irradiation resistance.

\section{Compound addition of multiple alloying elements}

The effects of multiple alloying elements on the CoCrNi alloy are exhibited in three aspects: precipitation strengthening; grain refinement; thermal stability. The strengthening mechanisms are not independent of each other and may work simultaneously.

(1) Precipitation strengthening: The composite addition of $\mathrm{Al}$ and $\mathrm{Ti}$ was first studied for the destabilization of the supersaturated FCC into the $\mathrm{L}_{2}$ and $\sigma$ phases. The dominating phases of the alloys changed from 
$\mathrm{FCC}+\mathrm{L}_{2}$ to $\mathrm{FCC}+\mathrm{L}_{2}+\sigma$ and then to $\mathrm{FCC}+\sigma+\mathrm{L} 2_{1}$ as the $\mathrm{Al}$ and $\mathrm{Ti}$ concentrations increased ${ }^{[138]}$. A small amount of $\mathrm{Al}$ and $\mathrm{Ti}$ was added into the CoCrNi MEA to introduce the coherent precipitates ${ }^{[139-141]}$. Discontinuous precipitation colonies were observed along the grain boundaries. However, a continuous precipitation was observed in the inner grain. The spherical particles were uniformly distributed in the matrix. A combination of a YS of $2 \mathrm{GPa}$ and a tensile uniform elongation of $13 \%$ was achieved ${ }^{[141]}$. This is by far the best strength-ductility combination that this type of alloy can achieve. There were no deformation twins in the CoCrNi-AlTi alloys during deformation. Slone et al. ${ }^{[61]}$ revealed the microscopic mechanism of the $\mathrm{Al}$ inhibiting twinning formation.

In addition to the CoCrNi-AlTi alloy, the CoCrNi-AlMo ${ }^{[142]}$ and $\mathrm{CoCrNi}^{-\mathrm{NbV}^{[143]}}$ alloys also exhibited an improvement in their properties by precipitation strengthening.

(2) Grain refinement: As widely acknowledged, the cast alloys exhibits a coarse and anisotropic solidification microstructure with grains aligned along the heat-flow direction. This produces a bulky columnar structure outside and a relatively small region of coarse equiaxed grains in the center, resulting in inferior mechanical properties. In order to improve the performance of the as-cast alloy, Liu et al. ${ }^{[144]}$ successfully changed the grain shape (from columnar to equiaxed) and refined the grain size through Ti and $\mathrm{C}$ microalloying. The columnar to equiaxed transition resulted in a strong mutual affinity of Ti and C, leading to their build-up ahead of the solid-liquid interface, thereby enhancing the constitutional undercooling.

Zhang et al. ${ }^{[145]}$ found that $\mathrm{Al} / \mathrm{Ta}$ co-doping significantly refined the average equiaxed grain size. This implies slower grain coarsening kinetics in the $\mathrm{Al} / \mathrm{Ta}$ co-doping alloys, since large $\mathrm{Al} / \mathrm{Ta}$ atoms hinder the grain boundary migration during recrystallization.

(3) Thermal stability: The introduction of coherent precipitates also allows the CoCrNi alloy to be used in high-temperature fields ${ }^{[146-149]}$. An et al. ${ }^{[148]}$ studied the effect of $\mathrm{Al}$, Ti and Ta on the CoCrNi MEA. The composite addition not only promoted the $\gamma^{\prime}$ precipitation but also increased the stability of the $\gamma^{\prime}$ phase. Zhao et al. ${ }^{[149]}$ studied the thermal stability of the $\gamma^{\prime}$ nanoparticles. The coherent precipitates exhibited a sluggish coarsening behavior. Furthermore, the precipitate/matrix interfacial energy was reasonably small with a value of $\sim 4 \mathrm{~mJ} \cdot \mathrm{m}^{2}$. The diffusion coefficient of the alloy was one order of magnitude smaller than that of a conventional superalloy at the same temperature. Therefore, the excellent thermal stability of the nanoparticles was due to either the small interfacial energy or the combined effect of the slow diffusion and small interfacial energy.

Table 1 summarizes the RT tensile properties of the CoCrNi-based MEAs after alloying. Moreover, Figure 17 summarizes the mechanical properties of the CoCrNi-based MEAs obtained by different strengthening methods. A wider range of mechanical properties could be obtained by alloying. By modifying the microstructures of the alloys, better combinations of strength and ductility can be produced.

\section{OTHER MECHANICAL PROPERTIES}

\section{Dynamic shear properties}

The strength-ductility trade-off often occurs under quasi-static conditions. In this regard, it is necessary to study the experiments under dynamic shear conditions. Hopkinson bar experiments were used to study the dynamic shear behavior under impact shear loading ${ }^{[64]}$. The CoCrNi alloy shows superior dynamic shear properties over other metals and alloys investigated so far [Figure 18]. This could be attributed to the dynamic grain refinement, deformation twins and interactions between dislocations and twin boundaries. 
Table 1. Tensile properties of CoCrNi-based MEA at RT

\begin{tabular}{|c|c|c|c|c|c|c|}
\hline Element & Alloy & Phase & YS (MPa) & UTS (MPa) & TE (\%) & Ref. \\
\hline \multirow[t]{6}{*}{$\mathrm{Al}$} & CoCrNi-3Al & FCC & 342 & 790 & 69 & [52] \\
\hline & $\mathrm{Al}_{7.5} \mathrm{CoCrNi}$ & FCC & 770 & 1044 & 48 & [115] \\
\hline & $\mathrm{Al}_{2} \mathrm{CoCrNi}$ & FCC & 226.7 & 763.0 & 75.3 & [116] \\
\hline & $\mathrm{Al}_{4} \mathrm{CoCrNi}$ & FCC & 281.0 & 771.7 & 73.8 & [116] \\
\hline & $\mathrm{Al}_{6} \mathrm{CoCrNi}$ & FCC & 305.9 & 794.9 & 67.9 & [116] \\
\hline & $\mathrm{Al}_{0.3} \mathrm{CoCrNi}$ & FCC & $945 \pm 26$ & $1230 \pm 11$ & $37.5 \pm 0.5$ & [117] \\
\hline \multirow[t]{8}{*}{ Mo } & $\mathrm{CoCrNi}-3 \mathrm{Mo}$ & FCC & 733 & 1063 & 49 & {$[52]$} \\
\hline & $(\mathrm{CoCrNi})_{99} \mathrm{Mo}_{1}$ & FCC & $412 \pm 3$ & $914 \pm 5$ & $63 \pm 2$ & {$[120]$} \\
\hline & $(\mathrm{CoCrNi})_{97} \mathrm{Mo}_{3}$ & FCC & $475 \pm 5$ & $983 \pm 6$ & $69 \pm 2$ & {$[120]$} \\
\hline & $(\mathrm{CoCrNi})_{95} \mathrm{Mo}_{5}$ & FCC & $528 \pm 6$ & $1026 \pm 10$ & $47 \pm 3$ & {$[120]$} \\
\hline & $(\mathrm{CoCrNi})_{96} \mathrm{Mo}_{4}$ & $\mathrm{FCC}+\sigma$ & 488 & 902 & 35 & [121] \\
\hline & $\mathrm{CoCrNiMo}_{0.1}$ & FCC & $465 \pm 3$ & $912 \pm 12$ & $70.5 \pm 4.8$ & [122] \\
\hline & $\mathrm{CoCrNiMo}_{0.2}$ & $\mathrm{FCC}+\sigma$ & $644 \pm 5$ & $1071 \pm 17$ & $29.5 \pm 7.6$ & {$[120]$} \\
\hline & $(\mathrm{CoCrNi})_{93} \mathrm{Mo}_{7}$ & $\mathrm{FCC}+\sigma$ & $652 \pm 4$ & $1253 \pm 10$ & $44 \pm 2$ & [123] \\
\hline \multirow[t]{3}{*}{ w } & CoCrNi-3W & FCC & 1000.0 & 1300.0 & 38.0 & [53] \\
\hline & $(\mathrm{CoCrNi})_{99} \mathrm{~W}_{1}$ & $\mathrm{FCC}$ & $352 \pm 2$ & $810 \pm 6$ & $65 \pm 3$ & [125] \\
\hline & $(\mathrm{CoCrNi})_{97} \mathrm{~W}_{3}$ & FCC & $406 \pm 4$ & $899 \pm 6$ & $58 \pm 2$ & [125] \\
\hline B & CoCrNi-B800 & FCC & 940 & 1170 & 26 & {$[57]$} \\
\hline \multirow[t]{3}{*}{ C } & $(\mathrm{CoCrNi})_{99.25} \mathrm{~W}_{0.75}$ & FCC & 347 & 862 & 75 & [58] \\
\hline & CoCrNi-0.5C & $\mathrm{FCC}+\mathrm{Cr}_{23} \mathrm{C}_{6}$ & $595 \pm 20$ & $1182 \pm 33$ & $62 \pm 1.6$ & {$[129]$} \\
\hline & CoCrNi-1C & $\mathrm{FCC}+\mathrm{Cr}_{23} \mathrm{C}_{6}$ & $611 \pm 99$ & $1186 \pm 10$ & $50 \pm 1.3$ & [129] \\
\hline \multirow[t]{2}{*}{$N$} & CoCrNi-0.5N & $\mathrm{FCC}$ & $703 \pm 5$ & $1052 \pm 22$ & $66.9 \pm 1.2$ & [59] \\
\hline & CoCrNi-N & $\mathrm{Cr}_{2} \mathrm{~N}$ & 638 & 1050 & 54 & [133] \\
\hline \multirow[t]{4}{*}{$\mathrm{Si}$} & $\mathrm{CoCrNiSio}_{0.1}$ & FCC & 452 & 856 & 70 & {$[60]$} \\
\hline & $\mathrm{CoCrNiSi}_{0.2}$ & FCC & 476 & 926 & 83 & {$[60]$} \\
\hline & $\mathrm{CoCrNiSi}_{0.3}$ & FCC & 502 & 960 & 90 & {$[60]$} \\
\hline & $\mathrm{CoCrNiSi}_{0.2}$ & FCC & 330 & 841 & 67.1 & [137] \\
\hline \multirow[t]{3}{*}{ AlTi } & $(\mathrm{CoCrNi})_{94} \mathrm{Al}_{3} \mathrm{Ti}_{3}$ & FCC & 750 & 1300 & 45 & [139] \\
\hline & $(\mathrm{CoCrNi})_{94} \mathrm{Al}_{3} \mathrm{Ti}_{3}$ & $\mathrm{FCC}+\mathrm{L1}_{2}+\sigma$ & 1431 & 1683 & 15 & {$[140]$} \\
\hline & $(\mathrm{CoCrNi})_{94} \mathrm{Al}_{3} \mathrm{Ti}_{3}$ & $\mathrm{FCC}+\mathrm{L1}_{2}$ & 2000 & 2200 & 13 & [141] \\
\hline AIMo & $\mathrm{CoCrNiAl} \mathrm{O}_{0.1} \mathrm{Mo}_{0.1}$ & $\mathrm{FCC}$ & 1100 & 1304 & 22 & {$[142]$} \\
\hline $\mathrm{NbV}$ & $\mathrm{Co}_{22} \mathrm{Cr}_{22} \mathrm{Ni}_{48} \mathrm{Nb}_{4} \mathrm{~V}_{4}$ & $\mathrm{FCC}+\mathrm{DO}_{22}$ & 966.2 & 1261.7 & 29.1 & {$[143]$} \\
\hline AlTa & $(\mathrm{CoCrNi})_{92} \mathrm{Al}_{6} \mathrm{Ta}_{2}$ & FCC & $595 \pm 21$ & $998 \pm 2$ & $52 \pm 4$ & {$[145]$} \\
\hline
\end{tabular}

MEA: Medium-entropy alloy; RT: room temperature; YS: yield stress; UTS: ultimate tensile stress; TE: transmission electron; FCC: face-centered cubic.

At CTs, the grain refinement efficiency was higher, as was the density of twins, stacking faults, LomerCottrell locks and phase transformations. Therefore, the dynamic shear properties were more prominent at CTs.

\section{Creep}

Creep is a fundamental high-temperature property and is an important parameter for evaluating the safety and reliability of workpieces. Although the CoCrNi MEA has excellent performance at RT and CTs, its creep behavior has not been studied systematically so far. 


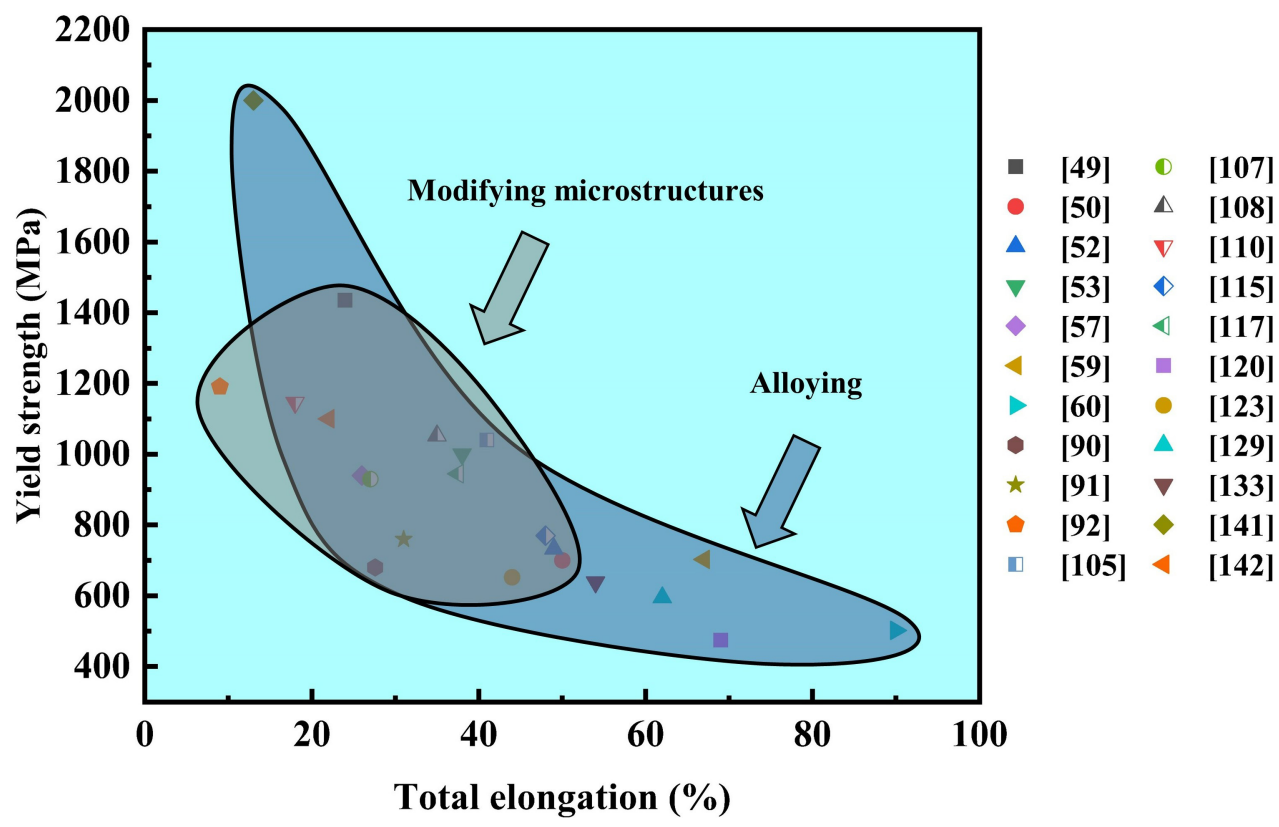

Figure 17. Mechanical properties of $\mathrm{CoCrNi}$-based alloys obtained by different strengthening methods. The gray area covers the alloys strengthened by modifying the microstructures. The dark blue area covers the alloys strengthened by optimizing the chemical composition $^{[4}$

Xie et al. ${ }^{[62]}$ investigated the tensile creep behavior of the CoCrNi MEA in a temperature range of 973-1073 K. Dislocation climb and lattice diffusion were found to be the dominant creep deformation mechanisms for this alloy. Cr-rich precipitates were observed along the grain boundaries in the fractured samples, indicating that the alloy decomposed during the high-temperature creep test. The uniaxial creep behavior of $\mathrm{CoCrNi}$ and other HEAs has not been extensively studied and many questions remain unanswered.

\section{Fracture toughness}

A structural component in service may fracture in three basic modes, i.e., mode I opening, mode II in-plane shear and mode III out-of-plane shear, and their combinations ${ }^{[111]}$. According to reports, at RT and liquid nitrogen temperature, the mode I fracture toughness of some FCC HEAs was comparable to that of the best stainless steels ${ }^{[\sigma, 27]}$. In-situ tensile tests at liquid nitrogen temperature of the $\mathrm{CoCrNi}$ alloy showed that a plastic zone exists in the vicinity of the crack tip, where the distorted deformation twins and dislocations offer additional strengthening mechanisms, and therefore the crack propagation is postponed ${ }^{[44]}$.

Yang et al.$^{[65]}$ investigated the impact toughness of the CoCrNi alloy at liquid helium temperature. The deformation activities and defect interactions were intensified by the high driving stresses and impact rate. A large number of primary and secondary twins were continuously embedded in the microstructure upon dynamic loading. This produced a large plastic deformation in the wide plastic zone ahead of the crack tip, causing work hardening and relieving local high stresses. Thus, it delayed the major shear band formation for crack propagation.

$\mathrm{Wu}$ et al. ${ }^{[159]}$ revealed the presence of shear bands (SBs) under impact loading [Figure 19]. When the SBs emerged, two types of secondary nano-twins formed around the developing SB. One type was in front of each advancing SB. The SB cuts through these nano-twins and often terminates there. The other type was formed inside the SBs, strengthening the SBs to reduce the tendency of micro-void nucleation in SBs and to 

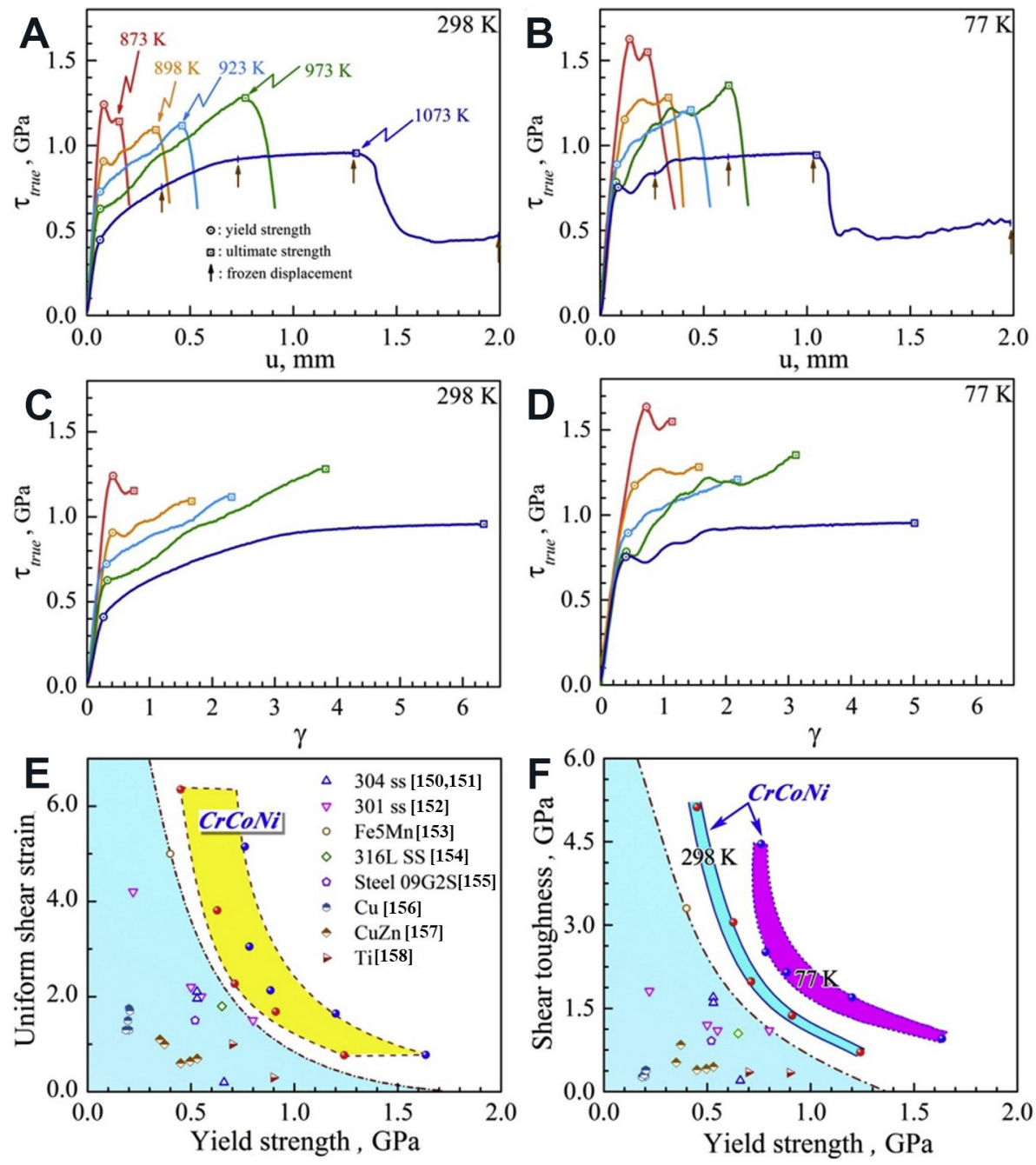

Figure 18. Shear stress-shear displacement curves of various microstructures at (A) RT and (B) CT. Shear stress-nominal shear strain curves of various microstructures for the experiments conducted at (C) RT and (D) CT. (E) Uniform dynamic shear strain vs. dynamic shear YS for the present MEA, along with data for other metals and alloys. (F) Impact shear toughness vs. dynamic shear YS for the present MEA, along with data for other metals and alloys ${ }^{[64,150-158]}$. MEA: Medium-entropy alloy; RT: room temperature; YS: yield stress; CT: cryogenic temperatures.
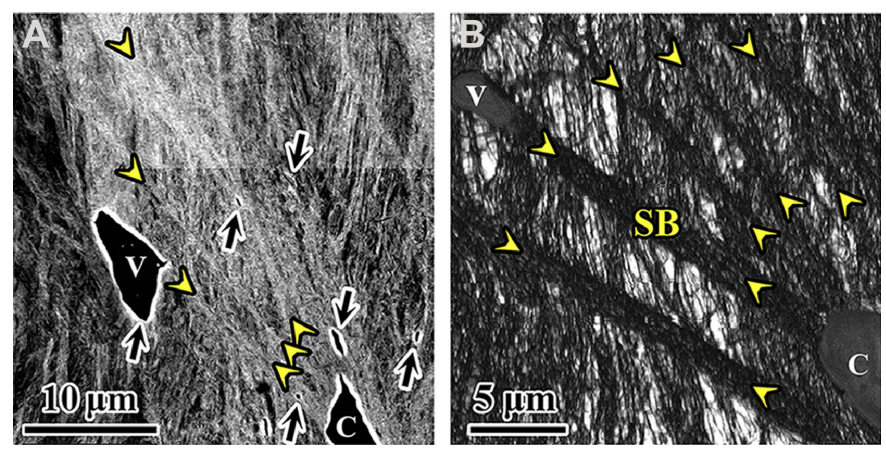

Figure 19. (A) Scanning electron microscopy image showing deformation features around the tip of the main crack (labeled by $C$ ) in a Charpy V-notch sample after $A_{k}$ testing at RT. (B) Close-up view showing the emission of SBs from the crack tip. Note the presence of a micro-void (labeled by $v$ ) on the advancing path of the $\mathrm{SB}^{[159]}$. SBs: Shear bands; RT: room temperature. 
delay the evolution from SBs into cracks. This self-toughening can reduce the void formation and crack propagation.

\section{SUMMARY AND PROSPECTS}

The CoCrNi alloy is a classical MEA with a single-phase FCC structure. Due to its low SFE, the alloy shows excellent comprehensive mechanical properties at RT and CTs. There has been extensive research on the mechanical properties of this alloy. Whether at RT or CTs, the deformation process of the alloy was mainly divided into three stages, namely, the dislocation, twinning and phase transformation stages. Compared with RT, the twins were activated at lower strain and the volume fraction of the HCP phase was higher at CTs.

A multitude of factors influenced the strength and ductility of the CoCrNi alloy, such as CRSS, SFE, SRO, $\mathrm{LD}$, preparation methods and processing routes. Thus, the CoCrNi alloy could be strengthened by modifying its microstructure and/or chemical structure. The microstructure could be modified in the scale range from crystal defects to grains, such as increasing the dislocation density, refining grains and forming heterogeneous structures. The optimization of the chemical structure was achieved by alloying. Precipitation and solution strengthening significantly improved the strength of the CoCrNi alloy by adding or doping substitutional elements and interstitial elements or even by the compound addition of multiple elements.

The CoCrNi alloy also showed an excellent performance in the dynamic shear test and the impact fracture toughness, surpassing all alloys reported so far. In addition to the mechanical properties, the physical properties need to be paid attention to by researchers. The new generation of materials requires the integration of structure and function. Therefore, under the condition of maintaining appropriate strength, the future development direction of the CoCrNi alloy can shift to functionality. The composition and microstructure of the alloy can be designed to make the alloy with optimized functions, such as high temperature stability and excellent resistance to oxidation and seawater corrosion.

\section{DECLARATIONS}

\section{Authors' contributions}

Conceptualization, investigation, writing - original draft: Xu D

Writing - review \& editing: Wang M, Li T, Wei X

Writing - review \& editing, supervision, funding acquisition: $\mathrm{Lu} \mathrm{Y}$

\section{Availability of data and materials}

Not applicable.

\section{Financial support and sponsorship}

This work was supported by the National Natural Science Foundation of China (Nos. 52001051, 51822402 and U20A20278), National Key Research and Development Program of China (Nos. 2019YFA0209901 and 2018YFA0702901), China Postdoctoral Science Foundation (Nos. 2020M670747 and 2021T140082), Liao Ning Revitalization Talents Program (No. XLYC1807047), and Fund of the State Key Laboratory of Solidification Processing in NWPU (No. SKLSP201902).

\section{Conflicts of interest}

All authors declared that there are no conflicts of interest. 


\section{Ethical approval and consent to participate}

Not applicable.

\section{Consent for publication}

Not applicable.

\section{Copyright}

(C) The Author(s) 2022.

\section{REFERENCES}

1. Cantor B, Chang I, Knight P, Vincent A. Microstructural development in equiatomic multicomponent alloys. Materials Science and Engineering: A 2004;375-377:213-8. DOI

2. Yeh J, Chen S, Lin S, et al. Nanostructured high-entropy alloys with multiple principal elements: novel alloy design concepts and outcomes. Adv Eng Mater 2004;6:299-303. DOI

3. Zhang Z, Han E, Xiang C. Irradiation behaviors of two novel single-phase bec-structure high-entropy alloys for accident-tolerant fuel cladding. J Mater Sci Mater Med 2021;84:230-8. DOI

4. Sun M, Liu X, Jiang W, et al. Grain boundary relaxation behavior and phase stability of AlCrTiV (x $=0,0.5$ and 1$)$ high-entropy alloys. Scr Mater 2021;204:114144. DOI

5. Muangtong P, Rodchanarowan A, Chaysuwan D, Chanlek N, Goodall R. The corrosion behaviour of CoCrFeNi-x $(\mathrm{x}=\mathrm{Cu}, \mathrm{Al}, \mathrm{Sn})$ high entropy alloy systems in chloride solution. Corros Sci 2020;172:108740. DOI

6. Gludovatz B, Hohenwarter A, Catoor D, Chang EH, George EP, Ritchie RO. A fracture-resistant high-entropy alloy for cryogenic applications. Science 2014;345:1153-8. DOI PubMed

7. Lu Y, Gao X, Jiang L, et al. Directly cast bulk eutectic and near-eutectic high entropy alloys with balanced strength and ductility in a wide temperature range. Acta Mater 2017;124:143-50. DOI

8. Gao X, Lu Y, Zhang B, et al. Microstructural origins of high strength and high ductility in an $\mathrm{AlCoCrFeNi}_{2.1}$ eutectic high-entropy alloy. Acta Mater 2017;141:59-66. DOI

9. Otto F, Yang Y, Bei H, George E. Relative effects of enthalpy and entropy on the phase stability of equiatomic high-entropy alloys. Acta Mater 2013;61:2628-38. DOI

10. Tang Z, Gao MC, Diao H, et al. Aluminum alloying effects on lattice types, microstructures, and mechanical behavior of highentropy alloys systems. JOM 2013;65:1848-58. DOI

11. Wang Z, Guo S, Liu CT. Phase selection in high-entropy alloys: from nonequilibrium to equilibrium. JOM 2014;66:1966-72. DOI

12. Guo S, Ng C, Liu C. Anomalous solidification microstructures in Co-free AlxCrCuFeNi2 high-entropy alloys. J Alloys Compd 2013;557:77-81. DOI

13. Roy U, Roy H, Daoud H, Glatzel U, Ray K. Fracture toughness and fracture micromechanism in a cast AlCoCrCuFeNi high entropy alloy system. Mater Lett 2014;132:186-9. DOI

14. Manzoni A, Daoud $\mathrm{H}$, Mondal $\mathrm{S}$, et al. Investigation of phases in $\mathrm{Al}_{23} \mathrm{Co}_{15} \mathrm{Cr}_{23} \mathrm{Cu}_{8} \mathrm{Fe}_{15} \mathrm{Ni}_{6}$ and $\mathrm{Al}_{8} \mathrm{Co}_{17} \mathrm{Cr}_{17} \mathrm{Cu}_{8} \mathrm{Fe}_{17} \mathrm{Ni}_{33}$ high entropy alloys and comparison with equilibrium phases predicted by Thermo-Calc. J Alloys Compd 2013;552:430-6. DOI

15. Singh S, Wanderka N, Kiefer K, Siemensmeyer K, Banhart J. Effect of decomposition of the Cr-Fe-Co rich phase of AlCoCrCuFeNi high entropy alloy on magnetic properties. Ultramicroscopy 2011;111:619-22. DOI PubMed

16. Pradeep K, Wanderka N, Choi P, Banhart J, Murty B, Raabe D. Atomic-scale compositional characterization of a nanocrystalline AlCrCuFeNiZn high-entropy alloy using atom probe tomography. Acta Mater 2013;61:4696-706. DOI

17. Singh S, Wanderka N, Murty B, Glatzel U, Banhart J. Decomposition in multi-component AlCoCrCuFeNi high-entropy alloy. Acta Mater 2011;59:182-90. DOI

18. Yang X, Chen SY, Cotton JD, Zhang Y. Phase stability of low-density, multiprincipal component alloys containing aluminum, magnesium, and lithium. JOM 2014;66:2009-20. DOI

19. Antonaglia J, Xie X, Tang Z, et al. Temperature effects on deformation and serration behavior of high-entropy alloys (HEAs). JOM 2014;66:2002-8. DOI

20. Wu Z, Bei H, Otto F, Pharr G, George E. Recovery, recrystallization, grain growth and phase stability of a family of FCC-structured multi-component equiatomic solid solution alloys. Intermetallics 2014;46:131-40. DOI

21. Wu Z, Bei H, Pharr G, George E. Temperature dependence of the mechanical properties of equiatomic solid solution alloys with facecentered cubic crystal structures. Acta Mater 2014;81:428-41. DOI

22. Yang S, Jiang M, Li H, Liu Y, Wang L. Assessment of Co-Cr-Ni ternary system by CALPHAD technique. Rare Metals 2012;31:7580. DOI

23. Omori T, Sato J, Shinagawa K, et al. Experimental determination of phase equilibria in the Co-Cr-Ni system. J Phase Equilib Diffus 2014;35:178-85. DOI

24. Chen J, Liu Y, Sheng G, Lei F, Kang Z. Atomic mobilities, interdiffusivities and their related diffusional behaviors in fcc Co-Cr-Ni alloys. J Alloys Compd 2015;621:428-33. DOI

25. Sales BC, Jin K, Bei H, et al. Quantum critical behavior in a concentrated ternary solid solution. Sci Rep 2016;6:26179. DOI PubMed PMC 
26. Zhao S, Osetsky Y, Zhang Y. Preferential diffusion in concentrated solid solution alloys: NiFe, NiCo and NiCoCr. Acta Mater 2017;128:391-9. DOI

27. Gludovatz B, Hohenwarter A, Thurston KV, et al. Exceptional damage-tolerance of a medium-entropy alloy CrCoNi at cryogenic temperatures. Nat Commun 2016;7:10602. DOI PubMed PMC

28. Miao J, Slone C, Smith T, et al. The evolution of the deformation substructure in a Ni-Co-Cr equiatomic solid solution alloy. Acta Mater 2017;132:35-48. DOI

29. Zhang Z, Sheng H, Wang Z, et al. Dislocation mechanisms and 3D twin architectures generate exceptional strength-ductilitytoughness combination in CrCoNi medium-entropy alloy. Nat Commun 2017;8:14390. DOI PubMed PMC

30. Yoshida S, Bhattacharjee T, Bai Y, Tsuji N. Friction stress and Hall-Petch relationship in CoCrNi equi-atomic medium entropy alloy processed by severe plastic deformation and subsequent annealing. Scr Mater 2017;134:33-6. DOI

31. Granberg F, Nordlund K, Ullah MW, et al. Mechanism of radiation damage reduction in equiatomic multicomponent single phase alloys. Phys Rev Lett 2016;116:135504. DOI PubMed

32. Laplanche G, Kostka A, Reinhart C, Hunfeld J, Eggeler G, George E. Reasons for the superior mechanical properties of mediumentropy CrCoNi compared to high-entropy CrMnFeCoNi. Acta Mater 2017;128:292-303. DOI

33. Zhang FX, Zhao S, Jin K, et al. Local structure and short-range order in a NiCoCr solid solution alloy. Phys Rev Lett 2017;118:205501. DOI PubMed

34. Jian W, Xie Z, Xu S, Su Y, Yao X, Beyerlein IJ. Effects of lattice distortion and chemical short-range order on the mechanisms of deformation in medium entropy alloy CoCrNi. Acta Mater 2020;199:352-69. DOI

35. Ali ML. Enhanced lattice distortion, yield strength, critical resolved shear stress, and improving mechanical properties of transitionmetals doped CrCoNi medium entropy alloy. RSC Adv 2021;11:23719-24. DOI

36. Tong $\mathrm{Y}$, Jin $\mathrm{K}$, Bei H, et al. Local lattice distortion in $\mathrm{NiCoCr}, \mathrm{FeCoNiCr}$ and FeCoNiCrMn concentrated alloys investigated by synchrotron X-ray diffraction. Mater Des 2018;155:1-7. DOI

37. Ding J, Yu Q, Asta M, Ritchie RO. Tunable stacking fault energies by tailoring local chemical order in CrCoNi medium-entropy alloys. Proc Natl Acad Sci US A 2018;115:8919-24. DOI PubMed PMC

38. Woo W, Jeong JS, Kim DK, et al. Stacking fault energy analyses of additively manufactured stainless steel $316 \mathrm{~L}$ and $\mathrm{CrCoNi}$ medium entropy alloy using in situ neutron diffraction. Sci Rep 2020;10:1350. DOI PubMed PMC

39. Woo W, Naeem M, Jeong J, et al. Comparison of dislocation density, twin fault probability, and stacking fault energy between CrCoNi and CrCoNiFe medium entropy alloys deformed at 293 and 140K. Materials Science and Engineering: A 2020;781:139224. DOI

40. Ikeda Y, Körmann F, Tanaka I, Neugebauer J. Impact of chemical fluctuations on stacking fault energies of $\mathrm{CrCoNi}$ and CrMnFeCoNi high entropy alloys from first principles. Entropy (Basel) 2018;20:655. DOI PubMed PMC

41. Huang H, Li X, Dong Z, et al. Critical stress for twinning nucleation in CrCoNi-based medium and high entropy alloys. Acta Mater 2018;149:388-96. DOI

42. Alhafez I, Ruestes CJ, Zhao S, Minor AM, Urbassek HM. Dislocation structures below a nano-indent of the CoCrNi medium-entropy alloy. Mater Lett 2021;283:128821. DOI

43. Slone C, Chakraborty S, Miao J, George E, Mills M, Niezgoda S. Influence of deformation induced nanoscale twinning and FCCHCP transformation on hardening and texture development in medium-entropy CrCoNi alloy. Acta Mater 2018;158:38-52. DOI

44. Feng X, Yang H, Fan R, et al. Heavily twinned CoCrNi medium-entropy alloy with superior strength and crack resistance. Materials Science and Engineering: A 2020;788:139591. DOI

45. Ma Y, Yang M, Yuan F, Wu X. Deformation induced hcp nano-lamella and its size effect on the strengthening in a CoCrNi mediumentropy alloy. J Mater Sci Mater Med 2021;82:122-34. DOI

46. Deng H, Wang M, Xie Z, et al. Enhancement of strength and ductility in non-equiatomic CoCrNi medium-entropy alloy at room temperature via transformation-induced plasticity. Materials Science and Engineering: A 2021;804:140516. DOI

47. Chen Y, Chen D, An X, et al. Unraveling dual phase transformations in a CrCoNi medium-entropy alloy. Acta Mater 2021;215:117112. DOI

48. Praveen S, Bae JW, Asghari-rad P, Park JM, Kim HS. Ultra-high tensile strength nanocrystalline CoCrNi equi-atomic medium entropy alloy processed by high-pressure torsion. Materials Science and Engineering: A 2018;735:394-7. DOI

49. Sathiyamoorthi P, Moon J, Bae JW, Asghari-rad P, Kim HS. Superior cryogenic tensile properties of ultrafine-grained CoCrNi medium-entropy alloy produced by high-pressure torsion and annealing. Scr Mater 2019;163:152-6. DOI

50. Wen R, You C, Zeng L, Wang H, Zhang X. Achieving a unique combination of strength and ductility in CrCoNi medium-entropy alloy via heterogeneous gradient structure. J Mater Sci 2020;55:12544-53. DOI

51. Yuan F, Cheng W, Zhang S, Liu X, Wu X. Atomistic simulations of tensile deformation in a CrCoNi medium-entropy alloy with heterogeneous grain structures. Materialia 2020;9:100565. DOI

52. Hu G, Zeng L, Du H, et al. Tailoring grain growth and solid solution strengthening of single-phase CrCoNi medium-entropy alloys by solute selection. J Mater Sci Mater Med 2020;54:196-205. DOI

53. Chen Y, Fang Y, Fu X, et al. Origin of strong solid solution strengthening in the CrCoNi-W medium entropy alloy. J Mater Sci Mater Med 2021;73:101-7. DOI

54. Jodi DE, Lee Y, Jang MH, et al. Investigation of plastic strain accommodation and recrystallization behavior in CoCrNiCu mediumentropy alloy. Mater Lett 2019;253:327-30. DOI

55. Lu W, Luo X, Yang Y, Huang B. Effects of $\mathrm{Nb}$ additions on structure and mechanical properties evolution of CoCrNi mediumentropy alloy. Materials Express 2019;9:291-8. DOI 
56. Feng X, Surjadi JU, Fan R, et al. Microalloyed medium-entropy alloy (MEA) composite nanolattices with ultrahigh toughness and cyclability. Mater Today 2021;42:10-6. DOI

57. Shi Y, Wang Y, Li S, Li R, Wang Y. Mechanical behavior in boron-microalloyed CoCrNi medium-entropy alloy studied by in situ high-energy X-ray diffraction. Materials Science and Engineering: A 2020;788:139600. DOI

58. Shang Y, Wu Y, He J, et al. Solving the strength-ductility tradeoff in the medium-entropy NiCoCr alloy via interstitial strengthening of carbon. Intermetallics 2019;106:77-87. DOI

59. Moravcik I, Hadraba H, Li L, Dlouhy I, Raabe D, Li Z. Yield strength increase of a CoCrNi medium entropy alloy by interstitial nitrogen doping at maintained ductility. Scr Mater 2020;178:391-7. DOI

60. Chang H, Zhang T, Ma S, et al. Novel Si-added CrCoNi medium entropy alloys achieving the breakthrough of strength-ductility trade-off. Mater Des 2021;197:109202. DOI

61. Slone C, Larosa C, Zenk C, George E, Ghazisaeidi M, Mills M. Deactivating deformation twinning in medium-entropy CrCoNi with small additions of aluminum and titanium. Scr Mater 2020;178:295-300. DOI

62. Xie D, Feng R, Liaw PK, Bei H, Gao Y. Tensile creep behavior of an equiatomic CoCrNi medium entropy alloy. Intermetallics 2020;121:106775. DOI

63. Agustianingrum MP, Lee U, Park N. High-temperature oxidation behaviour of CoCrNi medium-entropy alloy. Corros Sci 2020;173:108755. DOI

64. Ma Y, Yuan F, Yang M, Jiang P, Ma E, Wu X. Dynamic shear deformation of a CrCoNi medium-entropy alloy with heterogeneous grain structures. Acta Mater 2018;148:407-18. DOI

65. Yang M, Zhou L, Wang C, et al. High impact toughness of CrCoNi medium-entropy alloy at liquid-helium temperature. Scr Mater 2019;172:66-71. DOI

66. Uzer B, Picak S, Liu J, et al. On the mechanical response and microstructure evolution of NiCoCr single crystalline medium entropy alloys. Mater Res Lett 2018;6:442-9. DOI

67. Abuzaid W, Patriarca L. A study on slip activation for a coarse-grained and single crystalline CoCrNi medium entropy alloy. Intermetallics 2020;117:106682. DOI

68. Zhao S, Stocks GM, Zhang Y. Stacking fault energies of face-centered cubic concentrated solid solution alloys. Acta Mater 2017; 134:334-45. DOI

69. Niu C, LaRosa CR, Miao J, Mills MJ, Ghazisaeidi M. Magnetically-driven phase transformation strengthening in high entropy alloys. Nat Commun 2018;9:1363. DOI PubMed PMC

70. Zhang H, Yan H, Yu H, Ji Z, Hu Q, Jia N. The effect of Co and Cr substitutions for Ni on mechanical properties and plastic deformation mechanism of FeMnCoCrNi high entropy alloys. J Mater Sci Mater Med 2020;48:146-55. DOI

71. Yoshida S, Ikeuchi T, Bai Y, Tsuji N. Effect of cobalt-content on mechanical properties of non-equiatomic Co-Cr-Ni medium entropy alloys. Mater Trans 2020;61:587-95. DOI

72. Yan J, Fang W, Huang J, et al. Plastic deformation mechanism of CoCrxNi medium entropy alloys. Materials Science and Engineering: A 2021;814:141181. DOI

73. Lu C, Niu L, Chen N, et al. Enhancing radiation tolerance by controlling defect mobility and migration pathways in multicomponent single-phase alloys. Nat Commun 2016;7:13564. DOI PubMed PMC

74. Chen X, Wang Q, Cheng Z, et al. Direct observation of chemical short-range order in a medium-entropy alloy. Nature 2021;592:7126. DOI PubMed

75. Zhang R, Zhao S, Ding J, et al. Short-range order and its impact on the CrCoNi medium-entropy alloy. Nature 2020;581:283-7. DOI PubMed

76. Yin B, Yoshida S, Tsuji N, Curtin WA. Yield strength and misfit volumes of NiCoCr and implications for short-range-order. Nat Commun 2020;11:2507. DOI PubMed PMC

77. Praveen S, Bae JW, Asghari-rad P, Park JM, Kim HS. Annealing-induced hardening in high-pressure torsion processed CoCrNi medium entropy alloy. Materials Science and Engineering: A 2018;734:338-40. DOI

78. Schuh B, Völker B, Todt J, Kormout KS, Schell N, Hohenwarter A. Influence of annealing on microstructure and mechanical properties of a nanocrystalline CrCoNi medium-entropy alloy. Materials (Basel) 2018;11:662. DOI PubMed PMC

79. Weng F, Chew Y, Zhu Z, et al. Excellent combination of strength and ductility of CoCrNi medium entropy alloy fabricated by laser aided additive manufacturing. Addit Manuf 2020;34:101202. DOI

80. Niu P, Li R, Gan K, Yuan T, Xie S, Chen C. Microstructure, properties, and metallurgical defects of an equimolar CoCrNi medium entropy alloy additively manufactured by selective laser melting. Metall Mater Trans A 2021;52:753-66. DOI

81. Weng F, Chew Y, Zhu Z, et al. Influence of oxides on the cryogenic tensile properties of the laser aided additive manufactured CoCrNi medium entropy alloy. Compos B Eng 2021;216:108837. DOI

82. Wang J, Yang H, Ruan J, Wang Y, Ji S. Microstructure and properties of CoCrNi medium-entropy alloy produced by gas atomization and spark plasma sintering. J Mater Res 2019;34:2126-36. DOI

83. Moravcik I, Cizek J, Kovacova Z, et al. Mechanical and microstructural characterization of powder metallurgy CoCrNi medium entropy alloy. Materials Science and Engineering: A 2017;701:370-80. DOI

84. Sathiaraj G, Kalsar R, Kumar S, Suwas S, Skrotzki W. Evolution of texture during cold-rolling of a CrCoNi medium-entropy alloy. Mater Today Proc 2020;27:2147-51. DOI

85. Wu Y, Liu J, Bhatta L, Kong C, Yu H. Study of texture analysis on asymmetric cryorolled and annealed CoCrNi medium entropy alloy. Crystals 2020;10:1154. DOI

86. Dan Sathiaraj G, Skrotzki W, Pukenas A, et al. Effect of annealing on the microstructure and texture of cold rolled CrCoNi medium- 
entropy alloy. Intermetallics 2018;101:87-98. DOI

87. Sathiaraj GD, Skrotzki W, Immanuel RJ, et al. Microstructure and texture of cold rolled and recrystallized CrNoNi medium-entropy alloy. MSF 2018;941:833-8. DOI

88. Rémy L. The interaction between slip and twinning systems and the influence of twinning on the mechanical behavior of fcc metals and alloys. MTA 1981;12:387-408. DOI

89. Ding Q, Fu X, Chen D, et al. Real-time nanoscale observation of deformation mechanisms in CrCoNi-based medium- to high-entropy alloys at cryogenic temperatures. Mater Today 2019;25:21-7. DOI

90. Huang H, Wang J, Yang H, Ji S, Yu H, Liu Z. Strengthening CoCrNi medium-entropy alloy by tuning lattice defects. Scr Mater 2020;188:216-21. DOI

91. Liu Y, He Y, Cai S. Effect of gradient microstructure on the strength and ductility of medium-entropy alloy processed by severe torsion deformation. Materials Science and Engineering: A 2021;801:140429. DOI

92. Deng H, Xie Z, Zhao B, et al. Tailoring mechanical properties of a CoCrNi medium-entropy alloy by controlling nanotwin-HCP lamellae and annealing twins. Materials Science and Engineering: A 2019;744:241-6. DOI

93. Wang Y, Chen M, Zhou F, Ma E. High tensile ductility in a nanostructured metal. Nature 2002;419:912-5. DOI PubMed

94. Han B, Huang J, Zhu Y, Lavernia E. Strain rate dependence of properties of cryomilled bimodal 5083 Al alloys. Acta Mater 2006;54:3015-24. DOI

95. Zhao Y, Topping T, Bingert JF, et al. High tensile ductility and strength in bulk nanostructured nickel. Adv Mater 2008;20:3028-33. DOI

96. Han BO, Lavernia EJ, Lee Z, Nutt S, Witkin D. Deformation behavior of bimodal nanostructured $5083 \mathrm{Al}$ alloys. Metall and Mat Trans A 2005;36:957-65. DOI

97. Wu X, Yang M, Yuan F, et al. Heterogeneous lamella structure unites ultrafine-grain strength with coarse-grain ductility. Proc Natl Acad Sci U S A 2015;112:14501-5. DOI PubMed PMC

98. Lu K. Nanomaterials. Making strong nanomaterials ductile with gradients. Science 2014;345:1455-6. DOI PubMed

99. Wu X, Jiang P, Chen L, Yuan F, Zhu YT. Extraordinary strain hardening by gradient structure. Proc Natl Acad Sci U S A 2014;111:7197-201. DOI PubMed PMC

100. Wu XL, Jiang P, Chen L, Zhang JF, Yuan FP, Zhu YT. Synergetic strengthening by gradient structure. Mater Res Lett 2014;2:18591. DOI

101. Fang TH, Li WL, Tao NR, Lu K. Revealing extraordinary intrinsic tensile plasticity in gradient nano-grained copper. Science 2011;331:1587-90. DOI PubMed

102. Chen A, Liu J, Wang H, Lu J, Wang YM. Gradient twinned 304 stainless steels for high strength and high ductility. Materials Science and Engineering: A 2016;667:179-88. DOI

103. Wei Y, Li Y, Zhu L, et al. Evading the strength-ductility trade-off dilemma in steel through gradient hierarchical nanotwins. Nat Commun 2014;5:3580. DOI PubMed PMC

104. Yang M, Yan D, Yuan F, Jiang P, Ma E, Wu X. Dynamically reinforced heterogeneous grain structure prolongs ductility in a medium-entropy alloy with gigapascal yield strength. Proc Natl Acad Sci U S A 2018;115:7224-9. DOI PubMed PMC

105. Sathiyamoorthi P, Bae JW, Asghari-Rad P, Park JM, Kim JG, Kim HS. Effect of annealing on microstructure and tensile behavior of CoCrNi medium entropy alloy processed by high-pressure torsion. Entropy (Basel) 2018;20:849. DOI PubMed PMC

106. Gan B, Wheeler JM, Bi Z, Liu L, Zhang J, Fu H. Superb cryogenic strength of equiatomic CrCoNi derived from gradient hierarchical microstructure. J Mater Sci Mater Med 2019;35:957-61. DOI

107. Liu Y, He Y, Cai S. Gradient recrystallization to improve strength and ductility of medium-entropy alloy. J Alloys Compd 2021;853:157388. DOI

108. Sathiyamoorthi P, Asghari-rad P, Bae JW, Kim HS. Fine tuning of tensile properties in CrCoNi medium entropy alloy through cold rolling and annealing. Intermetallics 2019;113:106578. DOI

109. Wu X, Zhu Y. Heterogeneous materials: a new class of materials with unprecedented mechanical properties. Mater Res Lett 2017;5:527-32. DOI

110. Lu W, Luo X, Yang Y, Huang B. Hall-petch relationship and heterogeneous strength of CrCoNi medium-entropy alloy. Mater Chem Phys 2020;251:123073. DOI

111. Li W, Xie D, Li D, Zhang Y, Gao Y, Liaw PK. Mechanical behavior of high-entropy alloys. Prog Mater Sci 2021;118:100777. DOI PubMed PMC

112. Lu W, Luo X, Yang Y, Zhang J, Huang B. Effects of Al addition on structural evolution and mechanical properties of the CrCoNi medium-entropy alloy. Mater Chem Phys 2019;238:121841. DOI

113. Kim H, Lee D, Kim H, et al. The formation of B2-precipitate and its effect on grain growth behavior in aluminum-containing CoCrNi medium-entropy alloy. Mater Lett 2021;303:130481. DOI

114. Lee D, Jeong H, Lee K, Jeon JB, Park N. Precipitation and grain-boundary strengthening of Al-added CoCrNi medium-entropy alloys. Mater Lett 2019;250:127-30. DOI

115. Lee D, Agustianingrum MP, Park N, Tsuji N. Synergistic effect by Al addition in improving mechanical performance of CoCrNi medium-entropy alloy. J Alloys Compd 2019;800:372-8. DOI

116. Agustianingrum MP, Yoshida S, Tsuji N, Park N. Effect of aluminum addition on solid solution strengthening in CoCrNi mediumentropy alloy. J Alloys Compd 2019;781:866-72. DOI

117. Sathiyamoorthi P, Park JM, Moon J, et al. Achieving high strength and high ductility in Al0.3CoCrNi medium-entropy alloy through multi-phase hierarchical microstructure. Materialia 2019;8:100442. DOI 
118. Sathiyamoorthi P, Asghari-rad P, Park JM, et al. Exceptional cryogenic strength-ductility synergy in $\mathrm{Al}_{0.3} \mathrm{CoCrNi}_{\text {medium-entropy }}$ alloy through heterogeneous grain structure and nano-scale precipitates. Materials Science and Engineering: A 2019;766:138372. DOI

119. Wang Y, Liu B, Yan K, et al. Probing deformation mechanisms of a FeCoCrNi high-entropy alloy at 293 and $77 \mathrm{~K}$ using in situ neutron diffraction. Acta Mater 2018;154:79-89. DOI

120. Chang R, Fang W, Yan J, et al. Microstructure and mechanical properties of CoCrNi-Mo medium entropy alloys: experiments and first-principle calculations. J Mater Sci Mater Med 2021;62:25-33. DOI

121. He J, Makineni SK, Lu W, et al. On the formation of hierarchical microstructure in a Mo-doped NiCoCr medium-entropy alloy with enhanced strength-ductility synergy. Scr Mater 2020;175:1-6. DOI

122. Li N, Gu J, Gan B, Qiao Q, Ni S, Song M. Effects of Mo-doping on the microstructure and mechanical properties of CoCrNi medium entropy alloy. J Mater Res 2020;35:2726-36. DOI

123. Chang R, Fang W, Yu H, et al. Heterogeneous banded precipitation of (CoCrNi)93Mo7 medium entropy alloys towards strengthductility synergy utilizing compositional inhomogeneity. Scr Mater 2019;172:144-8. DOI

124. Wang J, Yang H, Huang H, Ruan J, Ji S. In-situ Mo nanoparticles strengthened CoCrNi medium entropy alloy. J Alloys Compd 2019;798:576-86. DOI

125. Chang R, Fang W, Bai X, et al. Effects of tungsten additions on the microstructure and mechanical properties of CoCrNi medium entropy alloys. J Alloys Compd 2019;790:732-43. DOI

126. Wu Z, Guo W, Jin K, Poplawsky JD, Gao Y, Bei H. Enhanced strength and ductility of a tungsten-doped CoCrNi medium-entropy alloy. J Mater Res 2018;33:3301-9. DOI

127. Jodi DE, Park N. Phase separation and its effect on atomic interactions in CoCrNiCu medium-entropy alloys. Mater Lett 2019;255:126528. DOI

128. Moravcik I, Gouvea L, Cupera J, Dlouhy I. Preparation and properties of medium entropy CoCrNi/boride metal matrix composite. $J$ Alloys Compd 2018;748:979-88. DOI

129. Moravcik I, Hornik V, Minárik P, et al. Interstitial doping enhances the strength-ductility synergy in a CoCrNi medium entropy alloy. Materials Science and Engineering: A 2020;781:139242. DOI

130. Byrnes M, Grujicic M, Owen W. Nitrogen strengthening of a stable austenitic stainless steel. Acta Metallurgica 1987;35:1853-62. DOI

131. Olsson CA, Hörnström SE. An AES and XPS study of the high alloy austenitic stainless steel 254 SMO $®$ tested in a ferric chloride solution. Corros Sci 1994;36:141-51. DOI

132. Roncery L, Weber S, Theisen W. Nucleation and precipitation kinetics of M23C6 and M2N in an Fe-Mn-Cr-C-N austenitic matrix and their relationship with the sensitization phenomenon. Acta Mater 2011;59:6275-86. DOI

133. Jodi DE, Choi N, Park J, Park N. Mechanical performance improvement by nitrogen addition in N-CoCrNi compositionally complex alloys. Metall Mater Trans A 2020;51:3228-37. DOI

134. Jodi DE, Park J, Park N. Precipitate behavior in nitrogen-containing CoCrNi medium-entropy alloys. Mater Charact 2019;157:109888. DOI

135. Moravcik I, Peighambardoust NS, Motallebzadeh A, et al. Interstitial nitrogen enhances corrosion resistance of an equiatomic CoCrNi medium-entropy alloy in sulfuric acid solution. Mater Charact 2021;172:110869. DOI

136. Yi H, Bi M, Yang K, Zhang B. Significant improvement the mechanical properties of CoCrNi alloy by tailoring a dual FCC-phase structure. Materials (Basel) 2020;13:4909. DOI PubMed PMC

137. Liu S, Lin W, Zhao Y, et al. Effect of silicon addition on the microstructures, mechanical properties and helium irradiation resistance of NiCoCr-based medium-entropy alloys. J Alloys Compd 2020;844:156162. DOI

138. Fang JYC, Liu WH, Luan JH, Jiao ZB. Phase stability and precipitation in L12-strengthened CoCrNi medium-entropy alloys at intermediate temperatures. J Phase Equilib Diffus 2021;42:781-93. DOI

139. Zhao Y, Yang T, Tong Y, et al. Heterogeneous precipitation behavior and stacking-fault-mediated deformation in a CoCrNi-based medium-entropy alloy. Acta Mater 2017;138:72-82. DOI

140. Yi H, Wei D, Xie R, Zhang Y, Kato H. A strategy for enhancing the mechanical property of the precipitation-strengthened mediumentropy alloy. Materials Science and Engineering: A 2021;819:141390. DOI

141. Du XH, Li WP, Chang HT, et al. Dual heterogeneous structures lead to ultrahigh strength and uniform ductility in a Co-Cr-Ni medium-entropy alloy. Nat Commun 2020;11:2390. DOI PubMed PMC

142. Liu X, Zhang M, Ma Y, et al. Achieving ultrahigh strength in CoCrNi-based medium-entropy alloys with synergistic strengthening effect. Materials Science and Engineering: A 2020;776:139028. DOI

143. Pan Y, Dong A, Zhou Y, et al. Enhanced strength-ductility synergy in a novel V-containing $\gamma^{\prime \prime}$-strengthened CoCrNi-based multicomponent alloy. Materials Science and Engineering: A 2021;816:141289. DOI

144. Liu X, Laplanche G, Kostka A, et al. Columnar to equiaxed transition and grain refinement of cast CrCoNi medium-entropy alloy by microalloying with titanium and carbon. $J$ Alloys Compd 2019;775:1068-76. DOI

145. Zhang D, Wang H, Zhang J, Xue H, Liu G, Sun J. Achieving excellent strength-ductility synergy in twinned NiCoCr medium-entropy alloy via A1/Ta co-doping. J Mater Sci Mater Med 2021;87:184-95. DOI

146. Slone C, George E, Mills M. Elevated temperature microstructure evolution of a medium-entropy CrCoNi superalloy containing A1,Ti. J Alloys Compd 2020;817:152777. DOI

147. Yi H, Wei D, Wang Y, et al. Hot deformation and dynamic recrystallization behavior of CoCrNi and (CoCrNi) $94 \mathrm{Ti} 3 \mathrm{Al} 3$ medium entropy alloys. Metals 2020;10:1341. DOI 
148. An N, Sun Y, Wu Y, et al. High temperature strengthening via nanoscale precipitation in wrought CoCrNi-based medium-entropy alloys. Materials Science and Engineering: A 2020;798:140213. DOI

149. Zhao Y, Yang T, Han B, et al. Exceptional nanostructure stability and its origins in the CoCrNi-based precipitation-strengthened medium-entropy alloy. Mater Res Lett 2019;7:152-8. DOI

150. Yang Y, Jiang F, Zhou B, Li X, Zheng H, Zhang Q. Microstructural characterization and evolution mechanism of adiabatic shear band in a near beta-Ti alloy. Materials Science and Engineering: A 2011;528:2787-94. DOI

151. Xue Q, Gray GT, Henrie BL, Maloy SA, Chen SR. Influence of shock prestraining on the formation of shear localization in 304 stainless steel. Metall and Mat Trans A 2005;36:1471-86. DOI

152. Xing J, Yuan F, Wu X. Enhanced quasi-static and dynamic shear properties by heterogeneous gradient and lamella structures in 301 stainless steels. Materials Science and Engineering: A 2017;680:305-16. DOI

153. Yuan F, Bian X, Jiang P, Yang M, Wu X. Dynamic shear response and evolution mechanisms of adiabatic shear band in an ultrafinegrained austenite-ferrite duplex steel. Mech Mater 2015;89:47-58. DOI

154. Xue Q, Gray GT. Development of adiabatic shear bands in annealed 316L stainless steel: Part I. Correlation between evolving microstructure and mechanical behavior. Metall and Mat Trans A 2006;37:2435-46. DOI

155. Pushkov V, Yurlov A, Bol'shakov A, Podurets A, Kal'manov A, Koshatova E. Study of adiabatic localized shear in metals by split Hopkinson pressure bar method. EPJ Web of Conferences 2010;10:00029. DOI

156. Pushkov V, Yurlov A, Podurets A, Tsibikov A, Novikov K, Pukhov M. Influence of preloading on formation of adiabatic localized shear in copper. EPJ Web of Conferences 2012;26:02001. DOI

157. Hofmann U, El-magd E. Behaviour of Cu-Zn alloys in high speed shear tests and in chip formation processes. Materials Science and Engineering: A 2005;395:129-40. DOI

158. Gu Y, Nesterenko VF. Dynamic behavior of HIPed Ti-6Al-4V. Int J Impact Eng 2007;34:771-83. DOI

159. Wu X, Yang M, Jiang P, et al. Deformation nanotwins suppress shear banding during impact test of CrCoNi medium-entropy alloy. Scr Mater 2020;178:452-6. DOI 